\title{
The in-plane elastic properties of hierarchical composite cellular materials: synergy of hierarchy, material heterogeneity and cell topologies at different levels
}

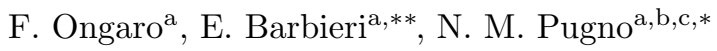 \\ ${ }^{a}$ School of Engineering and Materials Science, Queen Mary University of London, London, UK \\ ${ }^{b}$ Laboratory of Bio-Inspired and Graphene Nanomechanics, Department of Civil, Environmental and \\ Mechanical Engineering, University of Trento, Trento, Italy \\ ${ }^{c}$ Center for Materials and Microsystems, Fondazione Bruno Kessler, Povo (Trento), Italy
}

\begin{abstract}
The hierarchical organization of many biological materials plays a key role in their exceptional mechanical properties. Existing studies investigate how hierarchy affects the mechanical behavior of cellular materials and the vast majority of them assume empty cells. In reality, in numerous natural systems the cells are filled with fluids, fibers or other bulk materials to better resist external stimuli. Inspired by the highly efficiency of nature, this paper investigates the effects of adding hierarchy into a composite cellular material. Initially, the analytical expressions for the effective elastic moduli derived in the case of self-similarity reveal the system isotropy as for the not filled configuration. Then, from parametric analysis emerges a strong influence of the microstructure on the overall properties. We discovered that adding hierarchical levels to a filled cellular material can lead to a higher material specific stiffness only if the filler is stiffer than a critical value. Thus for classical cellular materials hierarchy is detrimental for the specific stiffness. In spite of this, for composite cellular solids an optimal number of hierarchical levels naturally emerges. In addition, numerical homogenization validates the analytical approach. Finally, the example of a hierarchical composite cellular material having different levels with different cell topologies is also considered. The present analysis provides an insight into the role of structural hierarchy on the in-plane elastic properties of composite cellular materials, as well as some possible ways to improve low-weight cellular structures by mixing different materials and varying the cell topology.
\end{abstract}

Keywords: composite, cellular material, Winkler model, linear elasticity, hierarchy, Finite Element Method, self-similarity

\footnotetext{
${ }^{*}$ Corresponding author

${ }^{* *}$ Co-corresponding author

Email addresses: f.ongaro@qmul.ac.uk (F. Ongaro), e.barbieri@qmul.ac.uk (E. Barbieri), nicola.pugno@unitn.it (N. M. Pugno)
} 


\section{Introduction}

It is well known that nature has developed a large number of ingenious solutions that served as a source of inspiration for scientists and engineers [1], [3].

In the literature, many works discuss this aspect. Among others, the pioneering textbook by Thompson [4] or, more recently, by Mattheck and Kubler [5], where the authors extract engineering principles from the structure of trees.

Nowadays, terms like biomimetics or bioinspiration [6], [7], [8] are commonly used to describe the new approach in chemistry, material science and engineering. That is, researchers study biological systems to find some useful principles to create e/o improve new materials and simplify many of our day-to-day functions.

Indeed, lessons learned from nature solved a variety of technical challenges in material science [14], architecture [15], aerodynamics and mechanical engineering [16]. For example, most are familiar with the Velcro, inspired by the way plant burrs stuck to animal fur [17], [18], the high performance swimsuits, modeled on the structure of shark skin to reduce drag in water [19] or the super adhesive fabrics that mimic the gecko foot configuration [20].

Differently from the engineer, nature has a relatively limited number of structural elements to choose, polymers, composites of polymers and ceramic particles [1]. Materials that certainly are not associated with strength, toughness, stiffness or durability. However, even with these restrictions, nature developed a wide range of systems with distinctive functions and remarkable mechanical properties that often surpass those of their components by orders of magnitude [21], as threes, skeletons, shells.

Even though it is still unknown how nature succeeded in doing this, some authors provided a number of possible strategies. Fratzl [8], for instance, suggests the two paradigms of growth and functional adaptation, that lead to the complex hierarchical architecture of natural materials. In particular, one advantage of hierarchical structuring is the multifunctionality. That is to say, a specific property, such as fracture toughness, can be tuned at different levels, independently of others properties, and adapted to the local needs [22], [23]. In other words, the exceptional mechanical behavior of biological systems is due to the functional adaptation of the structure at all levels of hierarchy [1].

In line with these theories, many studies and experimental observations on different natural materials, gecko foot, nacre shell, Armadillo armor, show that hierarchy is the nature's key of success [24].

In a system, hierarchy is reflected by several characteristics [22]. The first one, multiscality, is the coexistence of several structural levels with gradual transition in length scales ranging from nano to macro scale. The second, heterogeneity, is the presence of different properties at different levels. Also, a variety of designs are possible by changing type and configuration of the constituents [2] and, generally, the overall properties rarely reflect those of the constituents. Finally, anisotropy. As a consequence, many mathematical laws and material sciences' principles, that assume isotropy and homogeneity, must be carefully applied to hierarchical systems. General introductions on hierarchical biological materials include the recently published review articles [1], [21], [24], [22], [33].

Various authors have extensively studied structural hierarchy. Among them, Lakes [25] analyzes the hierarchical configuration of some natural materials, as fibrous composites and cellular solids, and of the man-made Eiffel Tower. It emerges that some desirable 
properties, like stress attenuation, superplasticity, increased toughness, are due to hierarchy. Other authors, like Pugno and Chen [26], [27], Haghpanah et al. [28], Ajdari et al. [29], Fan et al. [30], Taylor et al. [31] develop some numerical and theoretical models, force or energy based, to understand the role of hierarchy on the in-plane mechanical behavior of cellular solids. In particular, [26] and [28] focus on the elastic buckling while [27], [29], [30] obtain analytical expressions for the macroscopic elastic moduli. In addition, Bosia et al. [32] considers different hierarchical architectures of fiber bundles and, through multiscale calculations, proposed an analytical method to evaluate how hierarchy can affect the structural strength. Specifically, the study shows that, in the case of different types of fibers, the increase in the number of hierarchical levels leads to an improvement in the material strength. In the context of hierarchical materials with a selfsimilar microstructure, namely when the geometry is similar from one scale to another, several attempts have been made to model their mechanical behavior. [9] presents a finite element-based technique to evaluate the effective elastic moduli and scaling properties of two-dimensional materials containing self-similar multiscale voids/rigid inclusions whose distribution closely resemble the Serpinski-like carpet. The investigation suggests that increasing the levels of hierarchy provides an increase in the coefficient of anisotropy, leading to a mechanical behavior close to that of unidimensional materials. It also emerges that the scaling laws defining the transition between the properties belonging to different length of scale are power equations whose exponents are function of the inclusions/voids' dimension. A similar result is theoretically obtained in [12], that investigates self-similar media with different types of inhomogeneities and stress concentrators, such as pores, cracks, rigid inclusions. The proposed technique uses the concepts of the differential selfconsistent method [13] where it is assumed that equally-sized inhomogeneities does not interact directly. The interacting ones have different length of scale. The material is also represented as a sequence of homogenized continua of increasing scale, obtained in the average sense. An attempt to numerically solve boundary value problems for self-similar domains structured on a large number of scales is proposed in [11]. The authors, in particular, present a finite element procedure that employs modified shape functions to capture the complexity of the geometry at no additional computational cost. An extension of the concepts of classical Fracture Mechanics to cracks propagating in a self-similar regime is discussed in [10]. The analysis, in particular, focuses on scaling laws of fracture energy in brittle and quasi-brittle materials. It emerges that, independently of the material, the fracture energy is an exponential function with the exponent only related to the considered length of scale. Same considerations apply in the case of multiple cracks creating a self-similar pattern.

Inspired by the complex hierarchical organization of natural materials, the present paper focuses on the effects of adding hierarchy into a two-dimensional composite cellular material [34]. Namely, a cellular structure having the cells filled by a generic elastic material and a hierarchical architecture. In addition, the study investigates how hierarchy affect the macroscopic in-plane elastic moduli and whether it is possible to improve the specific stiffness by structural hierarchy, material mixing and varying cell topologies at different levels. Finally, as in [34], the Euler-Bernoulli beam on Winkler foundation elements model the microstructure at all levels.

A brief overview is of order. Initially, Section 2 focuses on a composite cellular material with a honeycomb microstructure and $n$ levels of hierarchy. The assumption that the length of scale of the sub-structure is fine enough to be negligible with respect to the 
super-structure [25] leads to the elastic constants in the continuum description. Then, Sections 3 and 4 present the comparison between the analytical and numerical approach, as well as the results of the parametric analysis to investigate the influence of the geometrical and mechanical microstructure parameters on the macroscopic properties. In particular, the analysis reveals that adding hierarchical levels to a cellular material can provide a higher material specific stiffness only if the filler is stiffer than a critical value. An optimal number of hierarchical levels also emerges. Conversely, for hollow cellular materials hierarchy is detrimental for the specific stiffness. To the authors' best knowledge, this is the first time to report such results.

\section{The hierarchical composite cellular material. Analytical model}

\subsection{Elastic constants}

A hierarchical material contains structural elements which themselves have structure [25]. Also, the hierarchical order of the material, $n$, can be defined as the number of levels of scale with recognized structure [25]. This paper deals with the in-plane analysis of a hierarchical composite cellular material having $n$ levels of hierarchy. That is to say, a material with $n$ hierarchical levels, a honeycomb-like architecture and the cells filled at each level (Fig. (1)). As in [34], a sequence of Euler-Bernoulli beams on Winkler foundation forming a periodic array of hexagonal cells simulates the underlying configuration at all levels. In accordance with [25], the length of scale of the cell walls' structure, the $(n-1)^{t h}$ level, is fine enough to be negligible with respect to the $n^{\text {th }}$ level. That is to say, the size of the structure of each cell wall is much smaller than the cell wall itself. As a consequence, a continuum having the elastic moduli derived in [34] approximates each cell arm.

First of all, let us focus on the first order hierarchical structure, $n=1$, of Figure (1). As it can be seen, it has hierarchy at one length of scale, like the composite honeycomb of [34] made by continuous cell walls, $n=0$. Accordingly, the effective Young's modulus and related Poisson's ratio in the $\mathbf{e}_{1}$ and $\mathbf{e}_{2}$ direction, $E_{1}^{(1)}, \nu_{12}^{(1)}$ and $E_{2}^{(1)}, \nu_{21}^{(1)}$ respectively, and shear modulus, $G^{(1)}$, are obtained by adopting the same approach of [34]. In particular, it emerges $E_{1}^{(1)}=E_{2}^{(1)} \equiv E^{(1)}, \nu_{12}^{(1)}=\nu_{21}^{(1)} \equiv \nu^{(1)}$ and

$$
\begin{gathered}
E^{(1)}=\frac{\left(13 K_{w}^{(1)}\left(1-\nu^{(0) 2}\right)+16 \lambda^{(1)} E^{(0)}\left(51\left(1+\left(\lambda^{(1)}\right)^{2}\right) K_{w}^{(1)}\left(1-\nu^{(0) 2}\right)+208\left(\lambda^{(1)}\right)^{3} E^{(0)}\right)\right.}{4 \sqrt{3}\left(1-\nu^{(0) 2}\right)\left(271\left(1+\left(\lambda^{(1)}\right)^{2}\right) K_{w}^{(1)}\left(1-\nu^{(0) 2}\right)+208\left(\lambda^{(1)}+3\left(\lambda^{(1)}\right)^{3}\right) E^{(0)}\right)}, \\
G^{(1)}=\frac{51\left(1+\left(\lambda^{(1)}\right)^{2}\right) K_{w}^{(1)}\left(1-\nu^{(0) 2}\right)+208\left(\lambda^{(1)}\right)^{3} E^{(0)}}{208 \sqrt{3}\left(1+\left(\lambda^{(1)}\right)^{2}\right)\left(1-\nu^{(0) 2}\right)} \\
\nu^{(1)}=\frac{67\left(1+\left(\lambda^{(1)}\right)^{2}\right) K_{w}^{(1)}\left(1-\nu^{(0) 2}\right)-208 \lambda^{(1)}\left(\left(\lambda^{(1)}\right)^{2}-1\right) E^{(0)}}{271\left(1+\left(\lambda^{(1)}\right)^{2}\right) K_{w}^{(1)}\left(1-\nu^{(0) 2}\right)+208 \lambda^{(1)}\left(1+3\left(\lambda^{(1)}\right)^{2}\right) E^{(0)}}
\end{gathered}
$$

with $E^{(0)}=E_{s}$ and $\nu^{(0)}=\nu_{s}$, on order, the Young's modulus and Poisson's ratio of the cell walls material, $K_{w}^{(1)}$ the Winkler foundation constant. To simplify the notation, $\lambda^{(1)}=h^{(1)} / \ell^{(1)}$, where $h^{(1)}$ and $\ell^{(1)}$ are, in turn, the thickness and the length of the cell arms. Moreover, taking into account the previous assumptions, the relations in (1), 
(2), (3) correspond to the elastic moduli of the cell walls in the case of hierarchy at two lengths of scale. Thus, the analysis in [34] still apply and substituting $E^{(1)}, \nu^{(1)}$ for $E^{(0)}$, $\nu^{(0)}$ and $\lambda^{(2)}=h^{(2)} / \ell^{(2)}, K_{w}^{(2)}$ for $\lambda^{(1)}, K_{w}^{(1)}$ in (1), (2), (3) provides the effective elastic constants $E_{1}^{(2)}=E_{2}^{(2)} \equiv E^{(2)}, \nu_{12}^{(2)}=\nu_{21}^{(2)} \equiv \nu^{(2)}, G^{(2)}$ of the second order hierarchical composite structure.

Analogous considerations leads to the elastic moduli of the $n^{\text {th }}$ level structure in the continuum form. That is to say, the Young's modulus, $E^{(n)} \equiv E_{1}^{(n)}=E_{2}^{(n)}$, shear modulus, $G^{(n)}$, Poisson's ratio, $\nu^{(n)} \equiv \nu_{12}^{(n)}=\nu_{21}^{(n)}$, are obtained by replacing $E^{(0)}, \nu^{(0)}$ and $\lambda^{(1)}, K_{w}^{(1)}$ by $E^{(n-1)}, \nu^{(n-1)}$ and $\lambda^{(n)}, K_{w}^{(n)}$ in (1), (2), (3). With obvious notation, $E^{(n-1)} \equiv E_{1}^{(n-1)}=E_{2}^{(n-1)}$ and $\nu^{(n-1)} \equiv \nu_{12}^{(n-1)}=\nu_{21}^{(n-1)}$ stand for the cell walls' Young's modulus, the first, and Poisson's ratio, the second, in the case of $n$ levels of hierarchy.

Finally, it emerges that the above elastic constants satisfy the classical relation

$$
G^{(i)}=\frac{E^{(i)}}{2\left(1+\nu^{(i)}\right)}, \quad i=1,2, \ldots, n,
$$

typical of the isotropic materials.

It should be noted that the composite microstructures analyzed in the present paper are represented by a sequence of elastic beams of unitary width. This assumption, in conjunction with the Winkler foundation to model the filler, enable us to obtain a closed form expression for the effective elastic constants, via an analytically tractable problem. Nevertheless, as a first approximation, the results can be extended to the case in which the cell walls are plates by assuming a not unitary width of the beams. Alternatively, to correctly capture the mechanical behavior and to obtain more accurate results, the beam should be replaced by plates. This leads to a mathematically less tractable problem and a closed form expression for the effective constants and constitutive equations could not be derived.

\subsection{The stiffness-to-density ratio}

Let us consider the first order hierarchical composite cellular structure of Figure (1). The cell walls, of density $\rho_{s}$, and the filling material, of density $\rho_{f}^{(1)}$, define every cell (Fig. (2)). From the rule of mixtures, the density of such composite configuration, $\rho^{(1)}$, is

$$
\rho^{(1)}=f^{(1)} \rho_{f}^{(1)}+\left(1-f^{(1)}\right) \rho_{s},
$$

with $f^{(1)}=V_{f}^{(1)} / V_{\text {tot }}^{(1)}$ the porosity, $V_{f}^{(1)}$ and $V_{\text {tot }}^{(1)}$, respectively, the volume of the filling material and of the entire cell. Simple geometrical considerations provide

$$
f^{(1)}=\frac{A_{f}^{(1)} b}{A_{t o t}^{(1)} b}=\frac{\sqrt{3}-2 \lambda^{(1)}}{\sqrt{3}},
$$

been $A_{f}^{(1)}$ and $A_{t o t}^{(1)}$, in turn, the area of the filling material and of the entire cell, $b$ the width and $\lambda^{(1)}$ the quantity previously defined. Replacing (6) into (5) leads to

$$
\rho^{(1)}=a^{(1)} \rho_{f}^{(1)}+b^{(1)} \rho_{s},
$$


where

$$
a^{(1)}=\frac{\sqrt{3}-2 \lambda^{(1)}}{\sqrt{3}}, \quad b^{(1)}=\frac{2 \lambda^{(1)}}{\sqrt{3}} .
$$

Assuming that the size of the microstructure of each cell wall is negligible with respect to the cell wall itself, (7) can also be treated as the cell walls' density in the case of two levels of hierarchy. Consequently, substituting $\lambda^{(2)}, \rho_{f}^{(2)}, \rho^{(1)}$ for $\lambda^{(1)}, \rho_{f}^{(1)}, \rho_{s}$ in (7), (8) gives the density of the hierarchical composite with two hierarchical levels.

Similarly, the density of the hierarchical composite cellular structure having $n$ levels of hierarchy is:

$$
\rho^{(n)}=a^{(n)} \rho_{f}^{(n)}+b^{(n)} \rho^{(n-1)},
$$

with

$$
a^{(n)}=\frac{\sqrt{3}-2 \lambda^{(n)}}{\sqrt{3}}, \quad b^{(n)}=\frac{2 \lambda^{(n)}}{\sqrt{3}},
$$

$\rho^{(n)}$ and $\rho^{(n-1)}$, respectively, the density of the filling material and of the cell walls at level $n$.

Accordingly, the stiffness-to-density ratio takes the form:

$$
\begin{aligned}
\frac{E^{(i)}}{\rho^{(i)}} & =\frac{E^{(i)}}{a^{(i)} \rho_{f}^{(i)}+b^{(i)} \rho^{(i-1)}}, \\
\frac{G^{(i)}}{\rho^{(i)}} & =\frac{E^{(i)}}{a^{(i)} \rho_{f}^{(i)}+b^{(i)} \rho^{(i-1)}}, \quad i=1,2, \ldots, n,
\end{aligned}
$$

been $E^{(i)}$ and $G^{(i)}$, in turn, the effective Young's modulus and shear modulus of the $i^{\text {th }}$ level hierarchical structure defined in Section 2.1.

\section{Discussion}

\subsection{Comparison between the analytical and numerical homogenization}

Finite element simulations on a computational model of the microstructure evaluate the prediction ability of the proposed modeling strategy. The study involves a three-level hierarchical composite cellular material having a honeycomb microstructure at all levels and such that the self-similar condition [26]

$$
\lambda^{(i)}=\lambda, \quad i=1,2,3,
$$

holds true. Specifically, a system with a self-similar property exhibits a statistically similar characteristic when examined both locally, at the level of individual entities, and globally, at the level of the whole system. In other words, the same general characteristic is independent of the scale at which the observation is made [41]. In biology, examples of self-similarity include the sticky foot of the Gecko, the trabecular bone, muscles and tendons [44], [46], [1], [45], composed by collagen fibers hierarchically arranged. Collagen, in particular, is a protein material with superior mechanical properties and provides itself an intriguing example of a hierarchical biological nano-material [45].

In terms of the material analyzed here, as in [34] the Euler-Bernoulli beam on Winkler foundation elements model the composite microstructure at all levels. The starting 
element of the hierarchical structure, the level (0) in Figure (1), has Young's modulus $E_{s}=79 \mathrm{GPa}$, Poisson's ratio $\nu_{s}=0.35$, density $\rho_{s}=2900 \mathrm{~kg} / \mathrm{m}^{3}$ (aluminum alloy) [3].

The assumption that the density of the filling material, $\rho_{f}^{(i)}$, is the same at all levels provides, similarly to (13),

$$
\rho_{f}^{(i)}=\rho_{f}=\alpha \rho_{s}, \quad i=1,2,3,
$$

been $\alpha$ a positive constant depending on the material inside the cells. In particular, assuming that a honeycomb cellular material fills the cells, as commonly happens in nature [3], leads to

$$
K_{w}^{(i)}=K_{w}=\frac{4 \sqrt{3}}{5} \alpha^{3} E_{s}, \quad i=1,2,3 .
$$

See Appendix A for further details.

As Figure (3) shows, the numerical simulations involve a $75 \times 50 \mathrm{~mm}$ rectangular domain discretized in an increasing number of hexagonal cells having gradually smaller length $\ell$ and thickness $h=0.1 \ell$. Also, $K_{w}=10^{-2} E_{s}$. Finally, the load conditions considered are the uniaxial compression in the $\mathbf{e}_{1}$ and $\mathbf{e}_{2}$ direction, Figure (3a) and Figure (3b) respectively, and pure shear, Figure (3c), simulated by forces acting at the unconstrained boundary nodes of the domain.

Table (1) illustrates the outcome of the analysis, in terms of the comparison between the theoretical and numerical values of the elastic moduli. It emerges that the analytical constants, derived from the expressions listed in Section 2.1, compare reasonably well with the numerical results, obtained by numerical homogenization [34]. In particular, the numerical solutions fastly converge to the analytical ones by increasing the number of cells that discretize the domain.

\subsection{The influence of the microstructure parameters in the macroscopic properties}

As it can be noted from the relations (1)-(3), the elastic constants of the approximated $i^{t h}$ order hierarchical structure, $i=1,2, \ldots, n$, are obviously related to the microstructure parameters. That is to say, the Young's modulus, $E^{(i-1)}$, and the Poisson's ratio, $\nu^{(i-1)}$, of the cell walls material, the ratio $\lambda^{(i)}=h^{(i)} / \ell^{(i)}$ between the thickness and the length of the cell arms, the Winkler foundation constant, $K_{w}^{(i)}$. Figures (4) and (5), based on a self-similar three-level hierarchical honeycomb as in Section 3.1, illustrate the influence of $\lambda^{(i)}$ and $K_{w}^{(i)}$ on the macroscopic elastic moduli.

When $K_{w}$ is fixed, Figures (4a), (4b) show that the stiffness-to-density ratio, $E^{(3)} / \rho^{(3)}$ and $G^{(3)} / \rho^{(3)}$, increases with increasing $\lambda$, namely, when the beam becomes thick. In particular, for $\lambda>0.1$ the increase is larger. As a matter of fact, the slope of the curves corresponding to $\lambda>0.1$ is bigger than that corresponding to $\lambda<0.1$. Furthermore, as expected, for an high value of $K_{w}\left(10^{-1} E_{s}\right)$ the initial values of $E^{(3)} / \rho^{(3)}$ and $G^{(3)} / \rho^{(3)}$ are higher than that occurring for small values of $K_{w}\left(10^{-3} E_{s}, 10^{-4} E_{s}\right)$. That is to say, an increase in the stiffness of the material filling the cells leads to a stiffer hierarchical composite cellular material. In terms of the Poisson ratio, Figure (4c) suggests that, for fixed $K_{w}$, an increase in $\lambda$ provides a decrease in $\nu^{(3)}$, that is more significant for small values of $K_{w}\left(10^{-3} E_{s}, 10^{-4} E_{s}\right)$. 
Regarding the influence of $K_{w}$ in the overall properties, $E^{(3)} / \rho^{(3)}, G^{(3)} / \rho^{(3)}$ and $\nu^{(3)}$, Figures (5a), (5b) suggest that, for fixed $\lambda$, to an increase of $K_{w}$ corresponds an high increase in the stiffness-to-density ratio. In particular, filling the cells with an elastic medium leads to an improvement in the specific stiffness, that is more evident in the case of thick beams. From Figure (5c) globally emerges that, for fixed $\lambda$, an increase in $K_{w}$ yields an increase in $\nu^{(3)}$, especially for high values of $\lambda(0.2,0.1)$. Also, the initial increase is followed by an almost horizontal line once $K_{w}$ reaches a specific value: $0.9 \times 10^{-1} E_{s}, 0.4 \times 10^{-1} E_{s}$ respectively for $\lambda=0.2$ and $\lambda=0.1$.

As stated, the stiffness of the material within the cells strongly affects the macroscopic properties of the hierarchical composite cellular material. One question that arises is how much it is possible to improve the specific stiffness of a standard hierarchical cellular material [26], [27] by filling its cells with a generic elastic medium. Table (2) presents the comparison between the stiffness-to-density ratio of a three-level standard hierarchical cellular material and of a three-level hierarchical composite cellular material. It emerges that the stiffness-to-density ratio of the filled-cells configuration, $E^{(3)} / \rho^{(3)}$ and $G^{(3)} / \rho^{(3)}$, is generally 1-3 times higher than that of the hollow one, $\left(E^{(3)} / \rho^{(3)}\right)_{k_{w}=0}$ and $\left(G^{(3)} / \rho^{(3)}\right)_{k_{w}=0}$. Obviously, increasing the stiffness of the filler, $K_{w}$, leads to an higher improvement.

Finally, differently from the standard hierarchical material [26], [27], [32], Figures (6) and (7) show that in the case of the filled-cells configuration an increase in the number of hierarchical levels provides an increase in the stiffness-to-density ratio. Nevertheless, to high values of $K_{w}\left(10^{-1} E_{s}, 10^{-2} E_{s}\right)$ corresponds an higher increase than that which occurs for a small value of $K_{w}\left(10^{-3} E_{s}\right)$. Conversely, for $K_{w}=10^{-4} E_{s}$, increasing the hierarchical levels lead to a decrease in $E^{(3)} / \rho^{(3)}$, as in the case of hollow configurations (Fig. (6)). Figure (7) also reveals that the stiffness-to-density ratio has an optimal value at level 4 for $K_{w}=10^{-1} E_{s}$, at level 3 for $K_{w}=10^{-2} E_{s}$ and at level 2 for $K_{w}=10^{-3} E_{s}$. It should be noted that in Figure (6) and (7) it is assumed $\lambda=0.1$. This not affect the outcome of the analysis, been the effect of $\lambda$ on the specific stiffness minimal (cfr. Figs. (4a), (4b)).

This result, in accordance with [32], indicates that both hierarchy and material heterogeneity are necessary to obtain improved stiffness. Also, it could be of great interest in practical applications as a strategy to design a more stiff bioinspired material via structural hierarchy and material mixing.

\subsection{Filled vs not-filled cells}

To thoroughly analyze how the material within the cells can affect the macroscopic mechanical behavior, this section initially deals with a traditional three-level hierarchical cellular material having a hexagonal microstructure at all levels. As in Sections 3.1 and 3.2 , the cell walls material has Young's modulus $E_{s}=79 \mathrm{GPa}$, Poisson's ratio $\nu_{s}=0.35$ and density $\rho_{s}=2900 \mathrm{~kg} / \mathrm{m}^{3}$ (aluminum alloy). Also, the self-similar conditions (13), (14), (15) still apply. Then, an elastic medium fills the cells of some levels and leaves empty the others, alternately. Specifically, the considered cases are listed in Table (3). The outcome of the analysis reveals that the macroscopic mechanical properties of a hierarchical composite cellular material are affected not only by the geometrical and mechanical parameters of the microstructure, $\lambda, E_{s}, \nu_{s}, K_{w}$, as emerged in Section 3.2, but also by having filled cells at one level rather than at another. 
As an example, let us consider the hierarchical composite structures of cases 2, 5, 7 of Table (3), that have filled cells at only one level. Respectively, the first, the second and the third. As Table (4) suggests, the resulting stiffness-to-density ratio, $E_{\text {case } i}^{(3)} / \rho_{\text {case } i}^{(3)}$ and $G_{\text {case } i}^{(3)} / \rho_{\text {case } i}^{(3)}$ have different values in the three cases. In particular, the hierarchical structure of case 2 is the least stiff while the configuration of case 7 has the higher stiffness. Accordingly, as pointed out in [23], in terms of macroscopic properties, the smaller the level, the less important is the presence of the filling material.

Focus now on cases $3,4,6$, that have filled cells at two levels, levels 1 and 2 , levels 1 and 3 , levels 2,3 , in turn. As emerges from Table (4), case 6 is the stiffer configuration while the elastic moduli of case 3 have the lowest values. As before, the macroscopic mechanical properties of a hierarchical composite structure are less affected by the presence of the filling material at small levels, as in case 3, rather than at larger levels, as in case 4 and 6 .

As a conclusion, the present study reveals that the effective elastic constants of a hierarchical composite cellular material can be optimized by carefully choosing the microstructure's parameters at each level.

\section{Different levels with different cell topologies}

As emerged in Section 3.2, the microstructure parameters generally affect the macroscopic elastic constants of a hierarchical composite cellular material. In Section 3.3 it also emerged that having filled cells at one level rather than at another leads to a hierarchical composite material with different elastic moduli. Namely, the mechanical properties of each level play an important role in the overall mechanical behavior. To further analyze such influence, this section treats a three-level hierarchical composite cellular material having different cell topologies at each level: the hexagonal, square and equilateral triangular, widely observed in natural and man-made cellular materials. In the considered configurations, listed in Table (5) and illustrated in Figure (8), the cell walls are assumed isotropic linear elastic, with Young's modulus $E_{s}=79 \mathrm{GPa}$, Poisson's ratio $\nu_{s}=0.35$, density $\rho_{s}=2900 \mathrm{~kg} / \mathrm{m}^{3}$ (aluminum alloy). Again, a honeycomb cellular material fills the cells at all levels and the conditions in (13), (14), (15) still apply. For more details, see Appendix B and C.

First of all, it should be noted that the hierarchical configurations analyzed in this section are not isotropic as those in Section 3. Thus, to provide a more complete description, Figures (9)-(12) illustrate the specific stiffness in different directions as a function of $K_{w}$. In particular, $(\cdot)_{0}^{(3)},(\cdot)_{30}^{(3)},(\cdot)_{45}^{(3)},(\cdot)_{60}^{(3)}$ stand, respectively, for the elastic moduli associated with the axis rotated counterclockwise by $0^{\circ}, 30^{\circ}, 45^{\circ}, 60^{\circ}$ from $\left(\mathbf{e}_{1}, \mathbf{e}_{2}\right)$.

A common feature in the plots of Figures (9)-(12) is the increase in the stiffness-todensity ratio for increasing $K_{w}$. Nevertheless, as expected, different results come from the six hierarchical configurations considered. Regarding Figure (9a), it emerges that cases 1 and 3, having a triangular microstructure at level 3, are the least performant in terms of $E_{0}^{(3)} / \rho^{(3)}$. Conversely, the hexagonal and square microstructures at level 3 , as in cases 2, 5 and 4, 6, respectively, provide high values of $E_{0}^{(3)} / \rho^{(3)}$. Accordingly, such configurations could be the best solution to obtain a hierarchical material with superior mechanical properties and minimum weight. Furthermore, in cases 1, 3, an increase in $K_{w}$ leads to a small increase in $E_{0}^{(3)} / \rho^{(3)}$ while in cases 2, 5 and 4,6 the increase is 
larger. Also, Figure (9b) shows that higher values of $G_{0}^{(3)} / \rho^{(3)}$ occur in cases 1,3 and 2, 5 rather than in cases 4,6 . In addition, Figure $(9 \mathrm{~b})$ reveals the existence of a value of $K_{w}, K_{w}^{*}=0.76 \times 10^{-3} E_{s}$, such that

$$
\begin{aligned}
& \left(\frac{G_{0}^{(3)}}{\rho^{(3)}}\right)_{\text {cases } 1,3}>\left(\frac{G_{0}^{(3)}}{\rho^{(3)}}\right)_{\text {cases } 2,5} \text { for } K_{w}<K_{w}^{*} \\
& \left(\frac{G_{0}^{(3)}}{\rho^{(3)}}\right)_{\text {cases } 1,3}<\left(\frac{G_{0}^{(3)}}{\rho^{(3)}}\right)_{\text {cases } 2,5} \text { for } K_{w}>K_{w}^{*}
\end{aligned}
$$

That is to say, small values of $K_{w}, K_{w}<K_{w}^{*}$, provide higher values of $G_{0}^{(3)} / \rho^{(3)}$ in cases 1,3 rather than in cases 2,5 . In contrast, in terms of $G_{0}^{(3)} / \rho^{(3)}$, cases 2,5 have superior stiffness than cases 1, 3 for high values of $K_{w}, K_{w}>K_{w}^{*}$.

As can be seen from Figures (10)-(12), the specific stiffness associated with the axis rotated by $30^{\circ}, 45^{\circ}, 60^{\circ}$ exhibits very low values if compared to the plots in Figure (9). The reason is that the square microstructure has a superior stiffness in the $\mathbf{e}_{1}$ and $\mathbf{e}_{2}$ directions, unlike in the other ones where the values are very low. This is due to the alignment of the cell walls in the loading direction. However, neglecting this aspect, in the case of Figure (10) the previous considerations still apply. That is to say, in terms of $E_{30}^{(3)} / \rho^{(3)}$ and $G_{30}^{(3)} / \rho^{(3)}$, cases 2, 5 and 4,6 have, on order, the highest and the lowest values. Conversely, regarding Figures (11)-(12), the hierarchical material having a triangular microstructure at level 3, namely the cases 1, 3, is the less stiff. Again, the hexagonal microstructure at level 3, cases 2, 5, makes the composite stiffer. Finally, focusing on Figure (13), it emerges that the effect of $K_{w}$ is minimal on the effective Poisson's ratio. As before, different values can be observed by considering different directions. In Figure (13a), (13c), (13d), it should be noted that cases 4, 6, having a square microstructure at level 3 , are not listed. The reason is that their Poisson's ratio vanishes (see Appendix B).

These findings could be useful to design/improve a new class of complex hierarchical materials with tailored parameters at each level. The plots in Figures (9)-(12) could also assist the selection of the cell topology for a given problem.

\section{Conclusions}

This paper, inspired by the complex hierarchical organization of many natural systems, investigates the effects of adding hierarchy into a two-dimensional composite cellular material subjected to in-plane loads. That is to say, a cellular material having filled cells. In particular, a sequence of Euler-Bernoulli beams on Winkler foundation models the microstructure at all levels.

Initially, the analysis deals with a composite cellular material having $n$ levels of hierarchy, a honeycomb microstructure and the cells filled at all levels. The assumption that the length of scale of the substructure is fine enough to be negligible with respect to the superstructure provides the macroscopic elastic constants. As expected, they are related to the geometrical and mechanical parameters of the microstructure at all levels. To investigate such influence, the example of a three-level structure is presented. It emerges 
that the macroscopic specific stiffness of the material is generally improved by increasing the stiffness of the filler. In particular, we discovered that adding hierarchical levels to a composite cellular material can lead to a higher specific stiffness only if the filling material is stiffer than a critical value. Thus for classical cellular materials hierarchy is detrimental for the specific stiffness. In spite of this, for filled cellular solids an optimal number of hierarchical level also emerges.

In addition, the analysis reveals that the elastic constant in the continuum description are affected not only by the aforementioned microstructure's parameters but also by having filled cells at one level rather than at another.

Then, the example of a three-level hierarchical orthotropic structure having different cell topologies, the hexagonal, square and equilateral triangular, is considered. From the investigation emerges that also the geometrical properties of the microstructure affect the effective elastic moduli of a hierarchical material. In particular, the hexagonal microstructure at level 3 is the best solution to obtain a material with superior stiffness and minimum weight.

Finally, finite element simulations verify the analytical approach.

In conclusion, this paper investigates the role of structural hierarchy, material heterogeneity and cell topology on the elastic constants of composite cellular materials. Some useful tools to create e/o improve complex hierarchical structures with tailored properties at each level are also included.

\section{Acknowledgement}

FO and EB are supported by the Queen Mary University of London Start-Up grant for new academics.

NMP is supported by the European Research Council (ERC StG Ideas 2011 BIHSNAM n. 279985, ERC PoC 2015 SILKENE nr. 693670), by the European Commission under the Graphene Flagship (WP14 Polymer Nanocomposites, n. 696656).

\section{References}

[1] Fratzl P., Weinkamer R., Nature's hierarchical materials, Mater. Sci., vol. 52, pp. 1263-1334, 2007.

[2] Barthelat F., Mirkhalaf M., The quest for stiff, strong and tough hybrid materials: an exhaustive exploration, J. R. Soc. Interface, vol. 10, pp. 1-11, 2013.

[3] Gibson L. J., Ashby M. F., Harley B. A., Cellular Materials in Nature and Medicine, Cambridge University Press , 2010.

[4] Thompson A. W., On growth and form - the complete revised edition, Dover Publications, 1992

[5] Mattheck C., Kubler H., The internal optimization of trees, Springer Verlag, Berlin, 1995.

[6] Sanchez C., Arribart H., Giraud Guille M. M., Biomimetism and bioinspiration as tools for the design of innovative materials and systems, Nature Materials, vol. 4, pp. 277-288, 2005.

[7] Vincent J. F. V., Bogatyreva O. A., Bogatyrev N. R, Bowyer A., Pahl A. K., Biomimetics: its practice and theory, J $R$ Soc Interface, vol. 3, pp. 471-482, 2006.

[8] Fratzl P., Biomimetic materials research: what can we really learn from nature's structural materials, J. R. Soc. Interface, vol. 4, pp. 637-642, 2007.

[9] Oshmyan V. G., Patlazhan S. A., Timan S. A., Elastic properties of Sierpinski-like carpets: Finiteelement-based simulation, Phys. Rev. E, vol. 64, 2001.

[10] Borodich F. M., Fractals and fractal scaling in fracture mechanics, Int. J. Frac., vol. 64, pp. 239-259, 1999.

[11] Soare M. A., Picu R. C., An approach to solving mechanics problems for materials with multiscale self-similar microstructure, Int. J. Sol. Struc., vol. 44, pp. 7877-7890, 2007. 
[12] Dyskin A. V., Effective characteristics and stress concentrations in materials with self-similar microstructure, Int. J. Sol. Struc., vol. 42, pp. 477-502, 2005.

[13] Salganik R. L., Mechanics of bodies with many cracks, Mech. Solids, vol. 8, pp. 135-143, 1973.

[14] Jeronimidis G., Atkins A. G., Mechanics of biological materials and structures - Nature's lessons for the engineer, J. Mech. Eng. Sci., vol. 209, pp. 221-235, 1995.

[15] Kemp M., Structural intuitions and metamorphic thinking in art, architecture and science, Metamorph - 9th Int. Architecture Exhibition Focus, pp. 30-43,Fondazione La Biennale di Venezia.

[16] Milwich M., Speck T., Speck O., Stegmaier T., Planck H., Biomimetics and technical textiles: solving engineering problems with the help of nature's wisdom., Am. J. Bot., vol. 93, pp. 12951305, 2006.

[17] Cohen Y. B., Biomimetics: mimicking and inspired-by biology, Proc. SPIE 5759, Smart Structures and Materials 2005: Electroactive Polymer Actuators and Devices (EAPAD), 2005).

[18] Jenkins C. H. M., Bio-Inspired Engineering, Momentum Press, 2012.

[19] Bixler G. D., Bhushan B., Bioinspired rice leaf and butterfly wing surface structures combining shark skin and lotus effects, Soft Matter, vol. 8, n. 44, pp. 11271-11284, 2012.

[20] Shah G. V., Sitti M., Modeling and Design of Biomimetic Adhesives Inspired by Gecko Foot-Hairs, Robotics and Biomimetics, pp. 873-878, 2004.

[21] Gibson, L. J., The hierarchical structure and mechanics of plant materials, J. R. Soc. Interface, vol. 9, pp. 2749-2766, 2012.

[22] Pan N., Exploring the significance of structural hierarchy in material systems - A review, Applied Physics Reviews, vol. 1, 2014.

[23] Gao H., Learning from Nature about Principles of Hierarchical Materials, Nanoelectronics Conference (INEC), 2010.

[24] Chen Q., Pugno N. M., Biomimetic mechanisms of natural hierarchical materials: a review, J. of the Mechanical Behavior of Biomedical Materials, vol. 19, pp. 3-33, 2013.

[25] Lakes R., Materials with structural hierarchy, Nature, vol. 361, pp. 511-515, 1993.

[26] Chen Q., Pugno N. M., In-plane elastic buckling of hierarchical honeycomb materials, Eur. J. Mech. A/Solids, vol. 34, pp. 120-129, 2012

[27] Pugno N. M., Chen Q., In-plane elastic properties of hierarchical cellular solids, Physics Engineering, vol. 10, pp. 3026-3031, 2011

[28] Haghpanah B., Papadopoulos J., Mousanezhad D., Hashemi H. N., Buckling of regular, chiral and hierarchical honeycombs under a general macroscopic stress state, Proc. R. Soc. A, vol. 470,2014

[29] Ajdari A., Jahromi B. H., Papadopoulos J., Hashemi H. N., Vaziri A., Hierarchical honeycombs with tailorable properties, Int. J. Solids Struct., vol. 49, pp. 1413-1419, 2012.

[30] Fan H. L., Jin F. N., Fang D. N., Mechanical properties of hierarchical cellular materials. Part I: Analysis, Composites Science and Technology, vol. 68, pp. 3380-3387, 2008.

[31] Taylor C. M., Smith C. W., Miller W., Evans K. E., The effects of hierarchy on the in-plane elastic properties of honeycombs, Int. J. Solids Struct., vol. 48, pp. 1330-1339, 2011.

[32] Bosia F., Abdalrahman T., Pugno N. M., Investigating the role of hierarchy on the strength of composite materials: evidence of a crucial synergy between hierarchy and material mixing, Nanoscale, vol. 4, pp. 1200-1207, 2012.

[33] Wegst U. G., Bai H., Saiz E., Tomsia A. P., Ritchie R. O., Bioinspired structural materials, nature materials, vol. 14, pp. 23-36, 2015

[34] Ongaro F., De Falco P., Pugno N. M., Barbieri E., Mechanics of filled cellular materials, Mech. Mater., vol. 97, pp. 26-47, 2016.

[35] Janco R, Solution Methods for Beam and Frames on Elastic Foundation Using the Finite Element Method, Mechanical Structures and Foundation Engineering, International scientific conference MSFE 2010

[36] Kumar R. S., McDowell D. L., Generalized continuum modeling of 2-D periodic cellular solids, Int. J. Solids Struct., vol. 41, pp. 7399-7422, 2004

[37] Bazant Z. P., Micropolar medium as model for buckling of grid frameworks, Proceedings of the 12th Midwestern Mechanics Conference, vol. 6, pp. 587-594, 1971

[38] Bazant Z. P., Christensen M., Analogy between micropolar continuum and grid frameworks under initial stress, Int. J. Solids Struct., vol. 8, pp. 327-346, 1972.

[39] Davini, C., Ongaro, F., A Homogenized Model for Honeycomb cellular materials, J. Elasticity, vol 104, pp. 205-226, 2011.

[40] Chen J. Y., Huang Y., Ortiz M., Fracture analysis of cellular materials: a strain gradient model, J. Mech. Phys. Solids, vol. 46, No. 5, pp. 789-828, 1998

[41] Katz J. S., The self-similar science system, Research Policy, vol. 28, pp. 501-517, 1999. 
[42] Behari J., Biophysical Bone Behavior: Principles and Applications, Wiley, 2009.

[43] Lipperman F., Ryvkin M., Fuchs M. B., Fracture toughness of two-dimensional cellular material with periodic microstructure, Int. J.Fract., vol. 146, pp. 279-290, 2007.

[44] Galvanetto U., Ferri Aliabadi M. H., Multiscale Modeling in Solid Machanics. Computational Approaches, Imperial College Press, 2010.

[45] Buehler M. J., Nanomechanics of collagen fibrils under varying cross-link densities: Atomistic and continuum studies, Journal of the Mechanical Behavior of Biomedical Materials I, vol. 41, pp. $59-67,2008$.

[46] Fratzl P., Collagen: Structure and Mechanics, an Introduction, Springer, 2008.

[47] Shen Z. L., Kahn H., Ballarini R., Eppell S. J., Viscoelastic Properties of Isolated Collagen Fibrils, Biophysical Journal, vol. 100, pp. 3008-3015, 2011. 
Figures and Tables

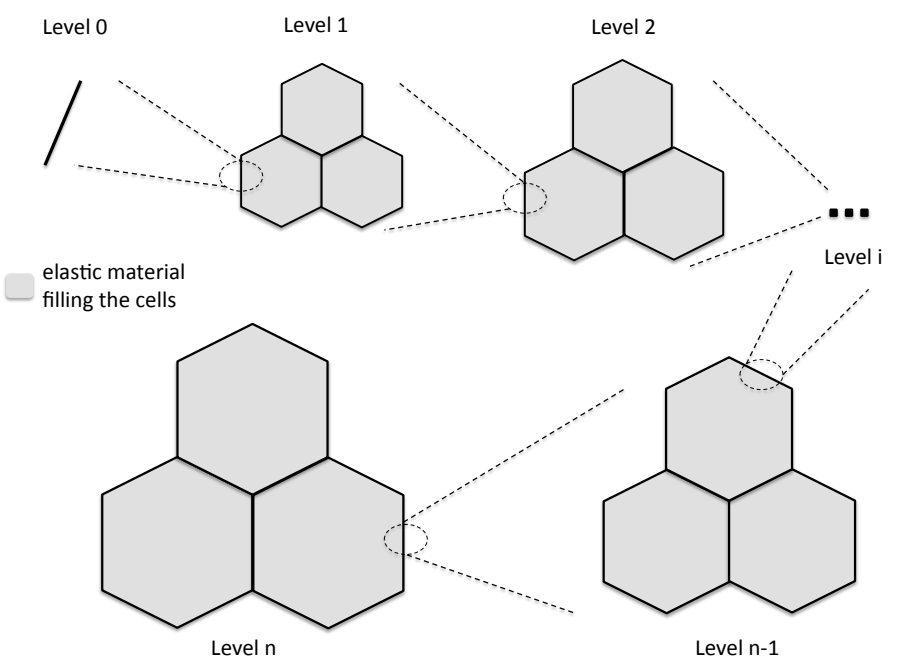

Figure 1: The hierarchical composite cellular material.

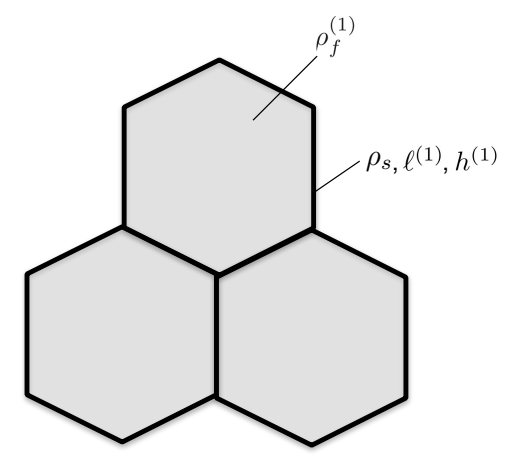

Figure 2: Density of the first order hierarchical structure. 

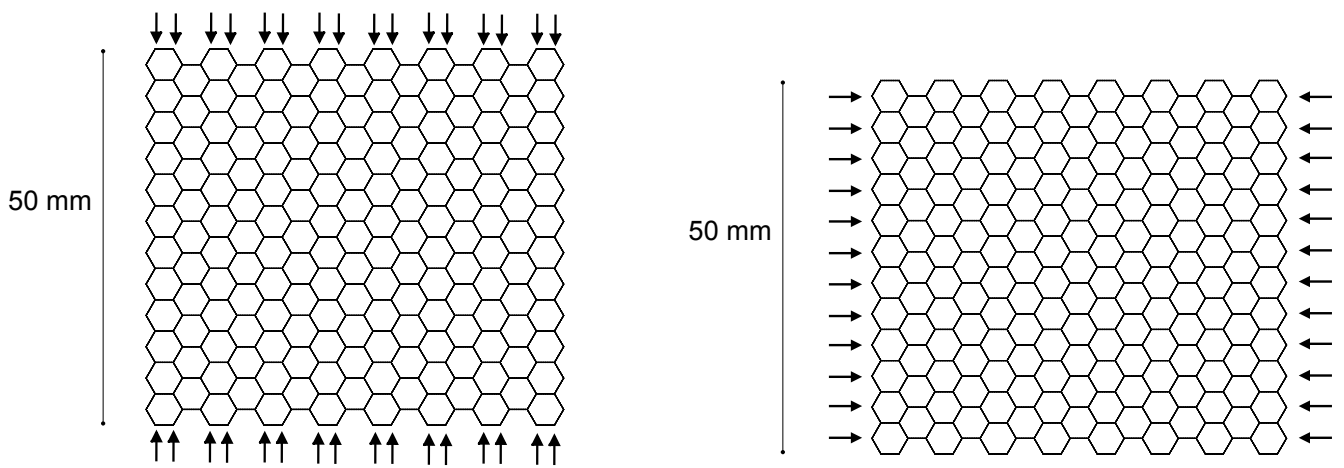

(a)

$75 \mathrm{~mm}$

(b)

$75 \mathrm{~mm}$

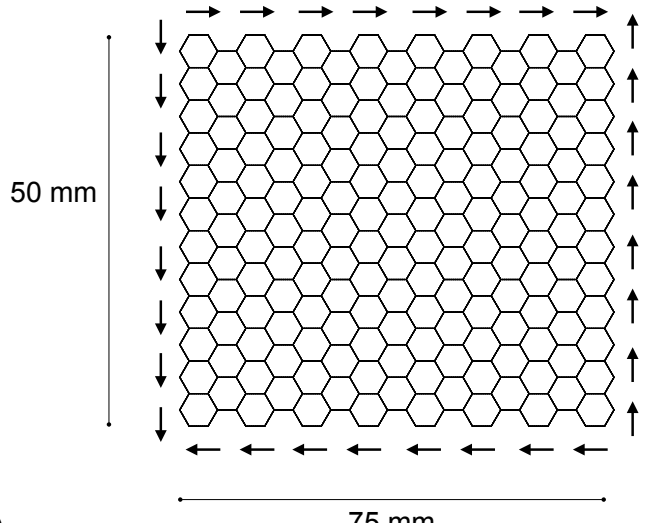

(c)

$75 \mathrm{~mm}$

Figure 3: Finite element implementation, the load conditions. (a) Uniaxial compression in the $\mathbf{e}_{1}$ direction, (b) Uniaxial compression in the $\mathbf{e}_{2}$ direction, (c) Pure shear.

Table 1: Comparison between the analytical and numerical approach, elastic moduli.

\begin{tabular}{ccccc}
\hline No. cells & $\ell(\mathrm{mm})$ & $E^{(3)}(\mathrm{GPa})$ & $\nu^{(3)}$ & $G^{(3)}(G P a)$ \\
\hline 10x7 & 5 & 3.40 & 0.30 & 0.30 \\
$50 \times 35$ & 1 & 3.08 & 0.31 & 0.30 \\
100x70 & 0.5 & 2.97 & 0.31 & 0.41 \\
200x140 & 0.25 & 2.97 & 0.32 & 0.54 \\
250x175 & 0.2 & 2.96 & 0.33 & 0.91 \\
400x280 & 0.125 & 2.96 & 0.33 & 0.92 \\
$500 \times 350$ & 0.1 & 2.94 & 0.33 & 0.92 \\
Analytical results & & 2.95 & 0.33 & 0.98 \\
\hline
\end{tabular}




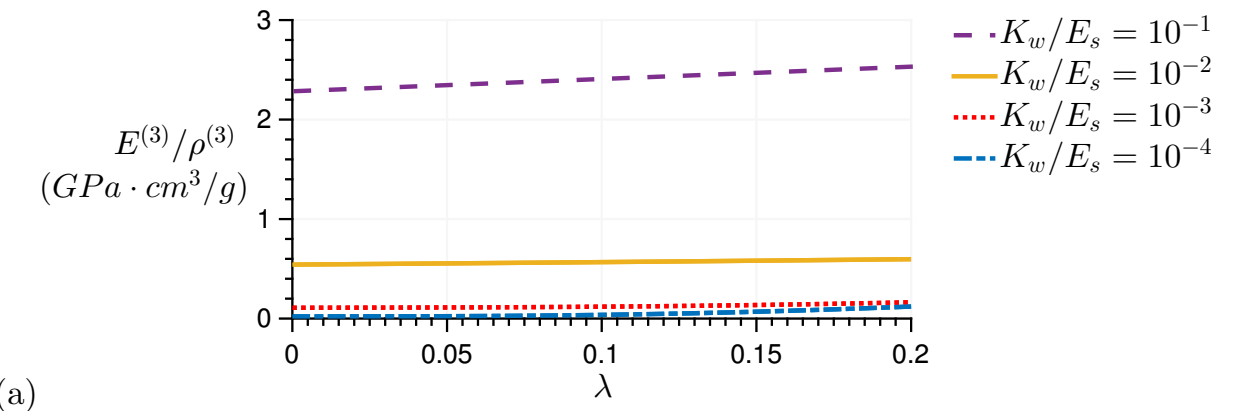

(a)

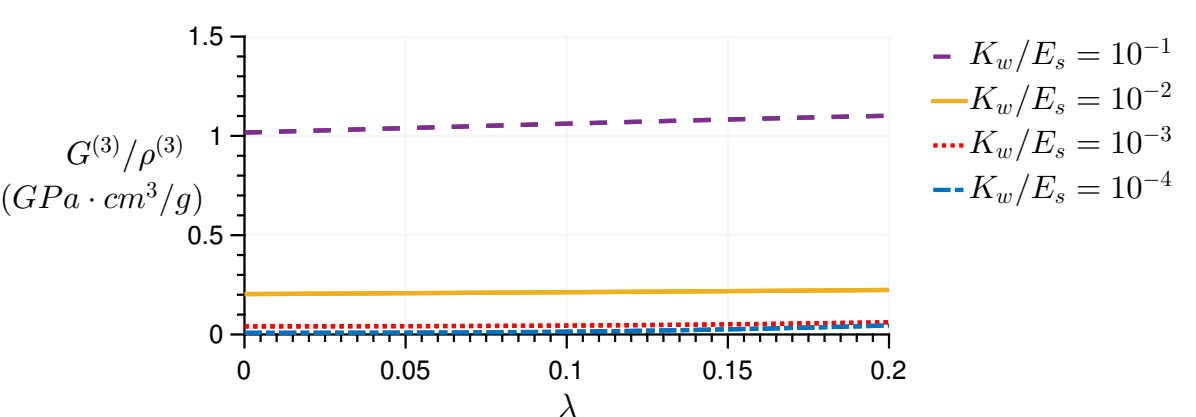

(b)

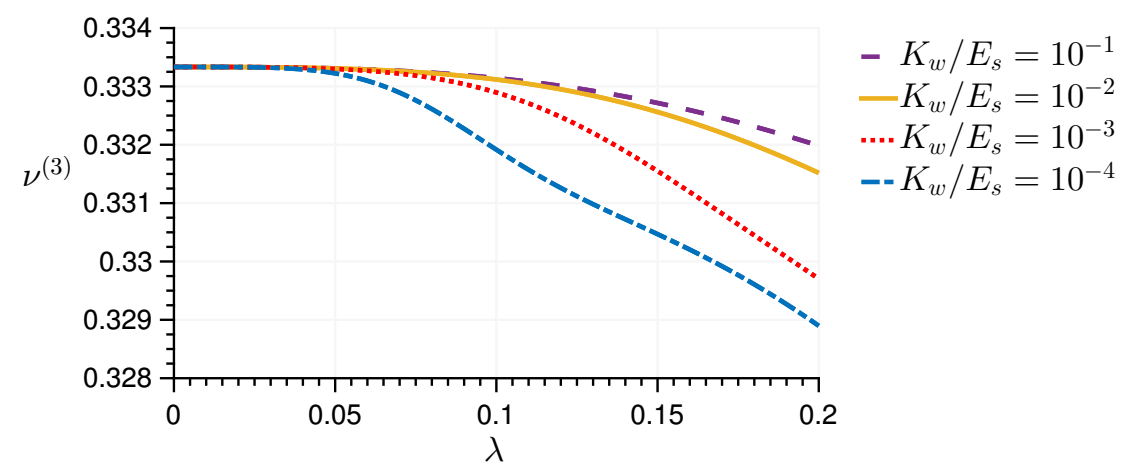

Figure 4: The influence of $\lambda$ in the three-level hierarchical composite cellular material. (a), (b) Stiffness-to-density ratio, (c) Poisson's ratio. 
(a)

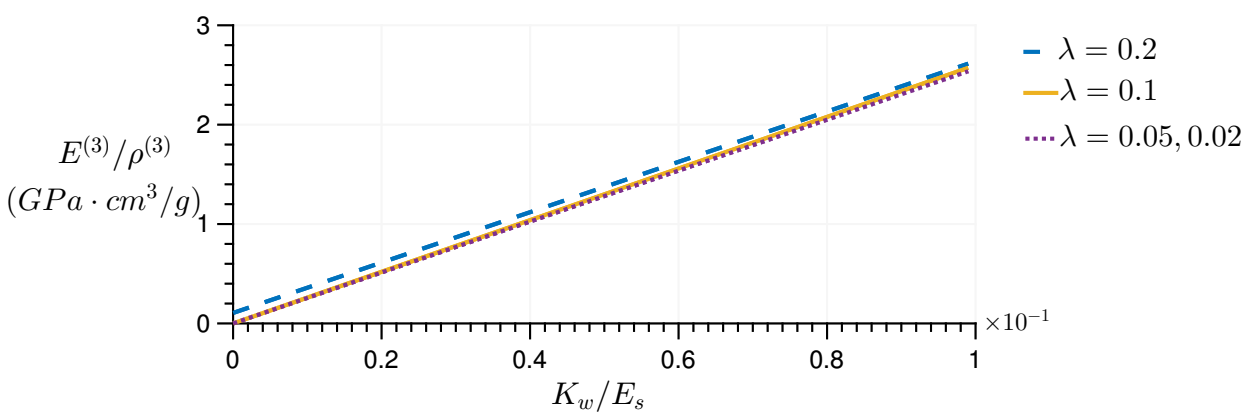

(b)

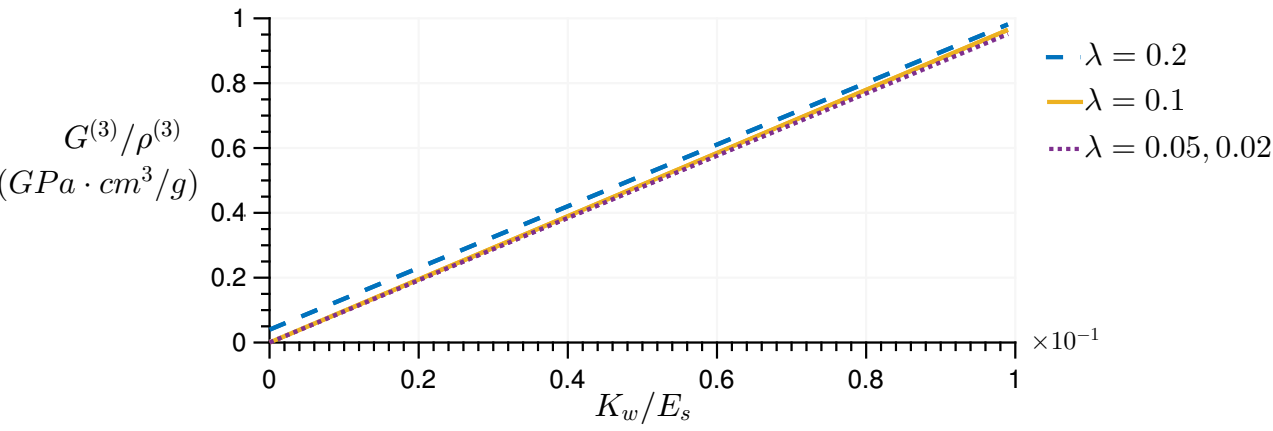

(c)

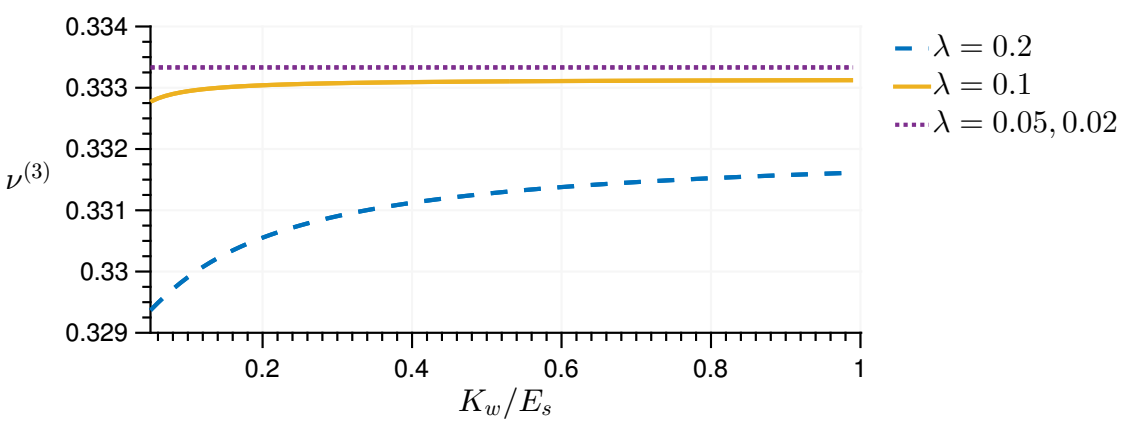

Figure 5: The influence of $K_{w}$ in the three-level hierarchical composite cellular material. (a), (b) Stiffness-to-density ratio, (c) Poisson's ratio.

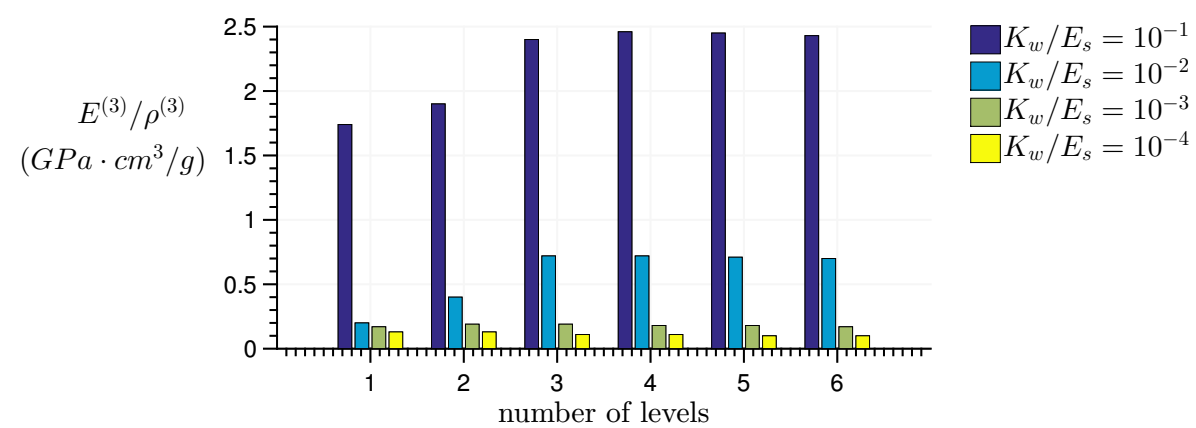

Figure 6: Stiffness-to-density ratio vs levels of hierarchy, with $\lambda=0.1$. 


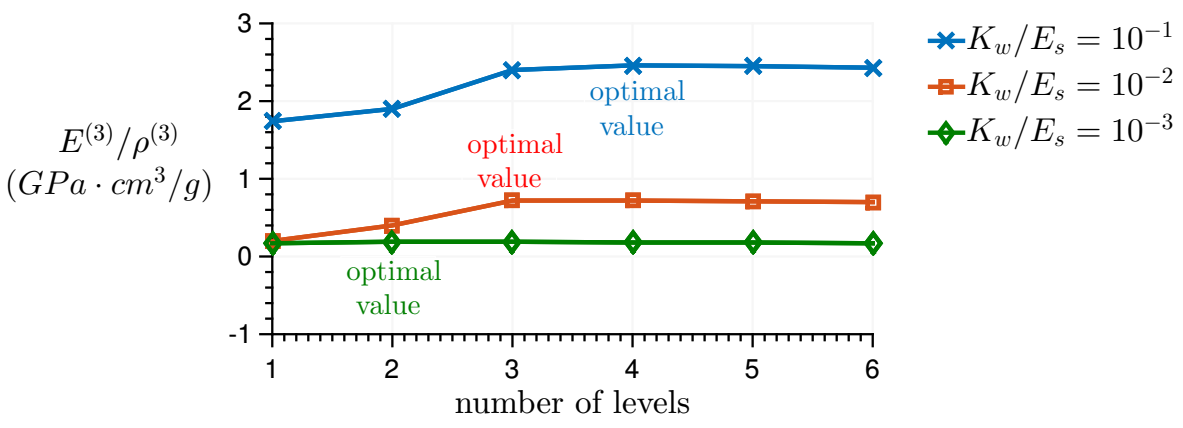

Figure 7: Stiffness-to-density ratio vs levels of hierarchy, optimal values in the case of $\lambda=0.1$.

Table 2: Comparison between a three-level hierarchical composite cellular material and a standard hierarchical cellular material. Stiffness-to-density ratio.

\begin{tabular}{|c|c|c|c|c|}
\hline \multicolumn{5}{|c|}{$\left(E^{(3)} / \rho^{(3)}\right) /\left(E^{(3)} / \rho^{(3)}\right)_{k_{w}=0}$} \\
\hline & $\lambda=0.2$ & $\lambda=0.1$ & $\lambda=0.05$ & $\lambda=0.02$ \\
\hline$K_{w}=10^{-4} E_{s}$ & 1.3 & 1.1 & 1.1 & 1.1 \\
\hline$K_{w}=10^{-3} E_{s}$ & 1.8 & 1.6 & 1.6 & 1.5 \\
\hline$K_{w}=10^{-2} E_{s}$ & 3.3 & 3.3 & 3.2 & 3.2 \\
\hline$K_{w}=10^{-1} E_{s}$ & 16.4 & 16.3 & 16.1 & 16 \\
\hline \multicolumn{5}{|c|}{$\left(G^{(3)} / \rho^{(3)}\right) /\left(G^{(3)} / \rho^{(3)}\right)_{k_{w}=0}$} \\
\hline & $\lambda=0.2$ & $\lambda=0.1$ & $\lambda=0.05$ & $\lambda=0.02$ \\
\hline$K_{w}=10^{-4} E_{s}$ & 1.4 & 1.1 & 1.1 & 1.1 \\
\hline$K_{w}=10^{-3} E_{s}$ & 1.8 & 1.8 & 1.7 & 1.6 \\
\hline$K_{w}=10^{-2} E_{s}$ & 3.8 & 3.6 & 3.6 & 3.4 \\
\hline$K_{w}=10^{-1} E_{s}$ & 19.7 & 19.3 & 19.3 & 19.1 \\
\hline
\end{tabular}

Table 3: Hierarchical configurations considered.

\begin{tabular}{cccc}
\hline & level 1 & level 2 & level 3 \\
\hline case 1 & not filled cells & not filled cells & not filled cells \\
case 2 & filled cells & not filled cells & not filled cells \\
case 3 & filled cells & filled cells & not filled cells \\
case 4 & filled cells & not filled cells & filled cells \\
case 5 & not filled cells & filled cells & not filled cells \\
case 6 & not filled cells & filled cells & filled cells \\
case 7 & not filled cells & not filled cells & filled cells \\
case 8 & filled cells & filled cells & filled cells \\
\hline
\end{tabular}


(a)

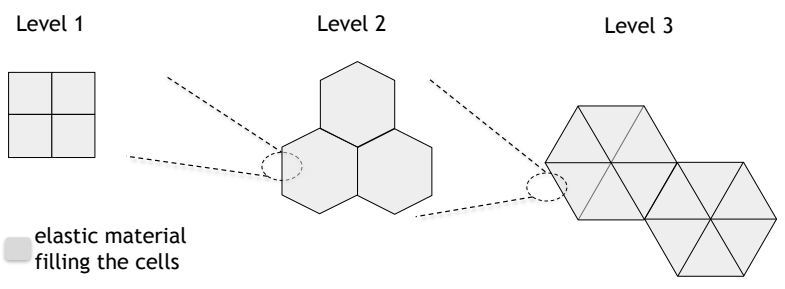

(b)
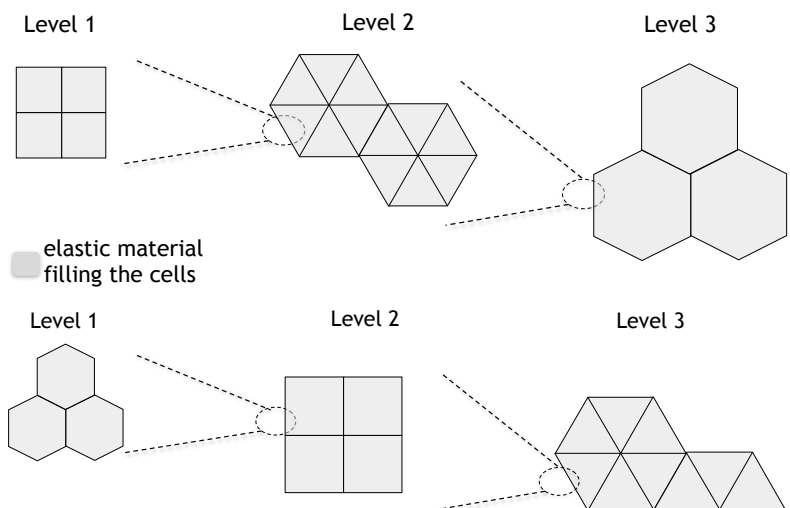

(c)

$$
\begin{aligned}
& \text { elastic material } \\
& \text { filling the cells }
\end{aligned}
$$

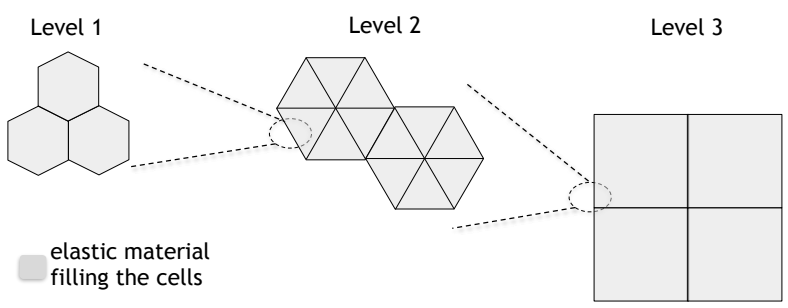

(d)

(e)
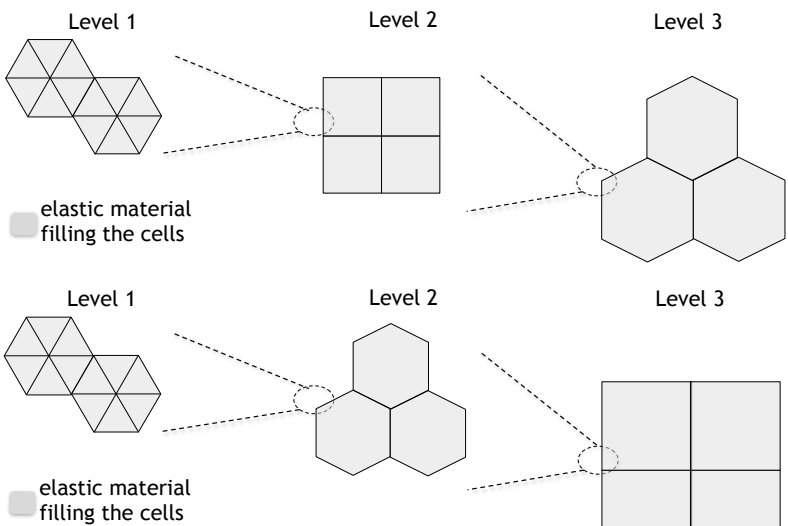

(f)

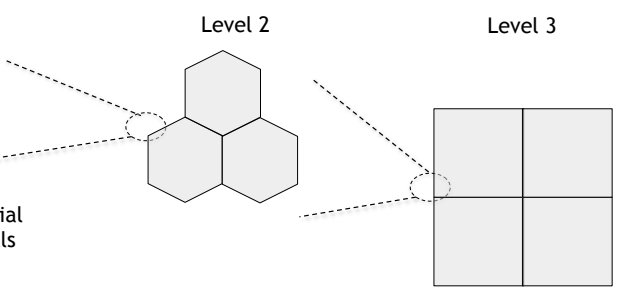

Figure 8: Different levels with different cell topologies: (a) Case 1, (b) Case 2, (c) Case 3, (d) Case 4, (e) Case 5, (f) Case 6. 


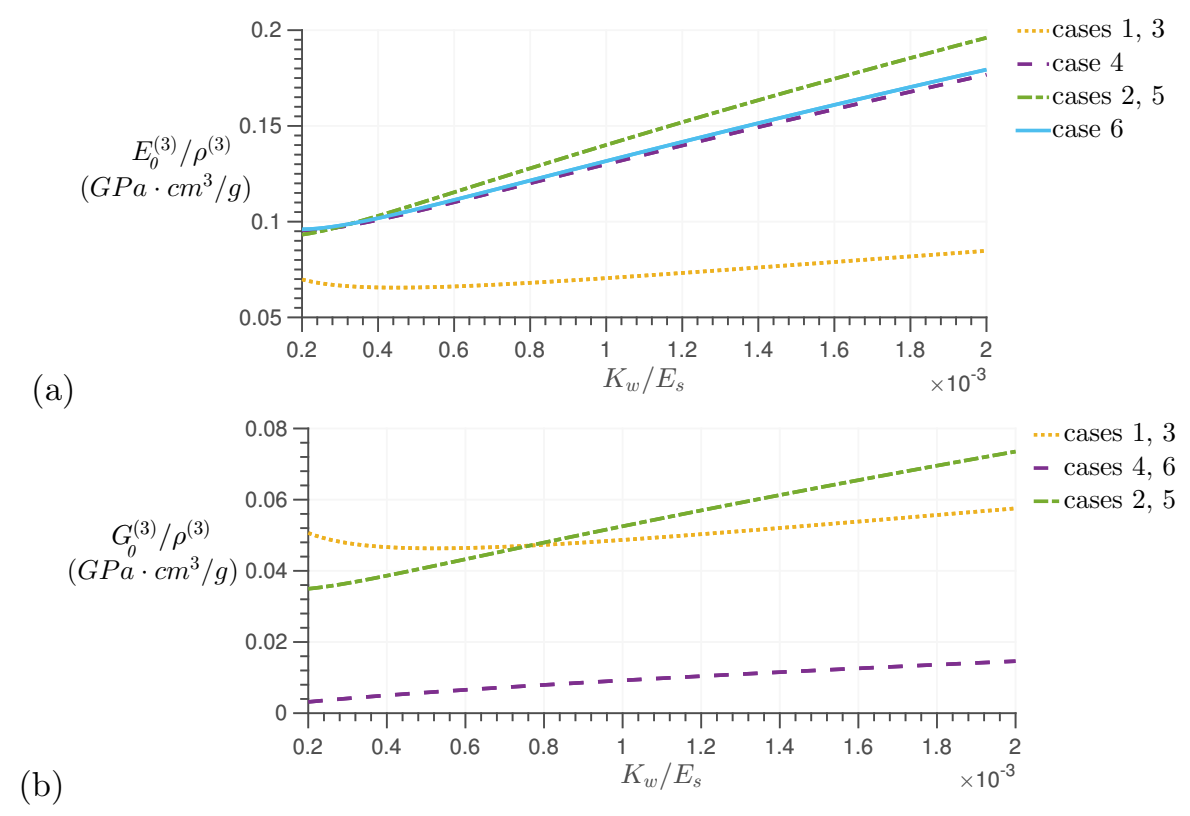

Figure 9: The influence of $K_{w}$ in the stiffness-to-density ratio associated with the axis $\left(\mathbf{e}_{1}, \mathbf{e}_{2}\right)$, with $\lambda=0.02:$ (a) Young's modulus, (b) Shear modulus.

Table 4: Filled vs not-filled. Elastic moduli, with $K_{w}=10^{-2} E_{s}$

\begin{tabular}{lcccc}
\hline \multicolumn{5}{c}{$E_{\text {case } i}^{(3)} / E_{\text {case } 1}^{(3)}$} \\
& $\lambda=0.2$ & $\lambda=0.1$ & $\lambda=0.05$ & $\lambda=0.02$ \\
case 2 & 1.21 & 1.20 & 1.18 & 1.18 \\
case 3 & 1.28 & 1.24 & 1.20 & 1.20 \\
case 4 & 1.92 & 1.86 & 1.86 & 1.85 \\
case 5 & 1.26 & 1.24 & 1.21 & 1.21 \\
case 6 & 2.70 & 2.70 & 2.50 & 2.50 \\
case 7 & 1.92 & 1.87 & 1.87 & 1.86 \\
case 8 & 3.30 & 3.30 & 3.20 & 3.20 \\
\hline & \multicolumn{5}{c}{$G_{\text {case } i}^{(3)} / G_{\text {case 1 }}^{(3)}$} \\
& $\lambda=0.1$ & $\lambda=0.05$ & $\lambda=0.02$ \\
case 2 & 1.25 & $\lambda=0.23$ & 1.20 & 1.20 \\
case 3 & 1.31 & 1.28 & 1.23 & 1.22 \\
case 4 & 2.10 & 2.00 & 1.92 & 1.90 \\
case 5 & 1.29 & 1.27 & 1.24 & 1.24 \\
case 6 & 3.10 & 3.00 & 2.90 & 2.90 \\
case 7 & 2.20 & 2.10 & 1.94 & 1.92 \\
case 8 & 3.80 & 3.60 & 3.60 & 3.40 \\
\hline
\end{tabular}




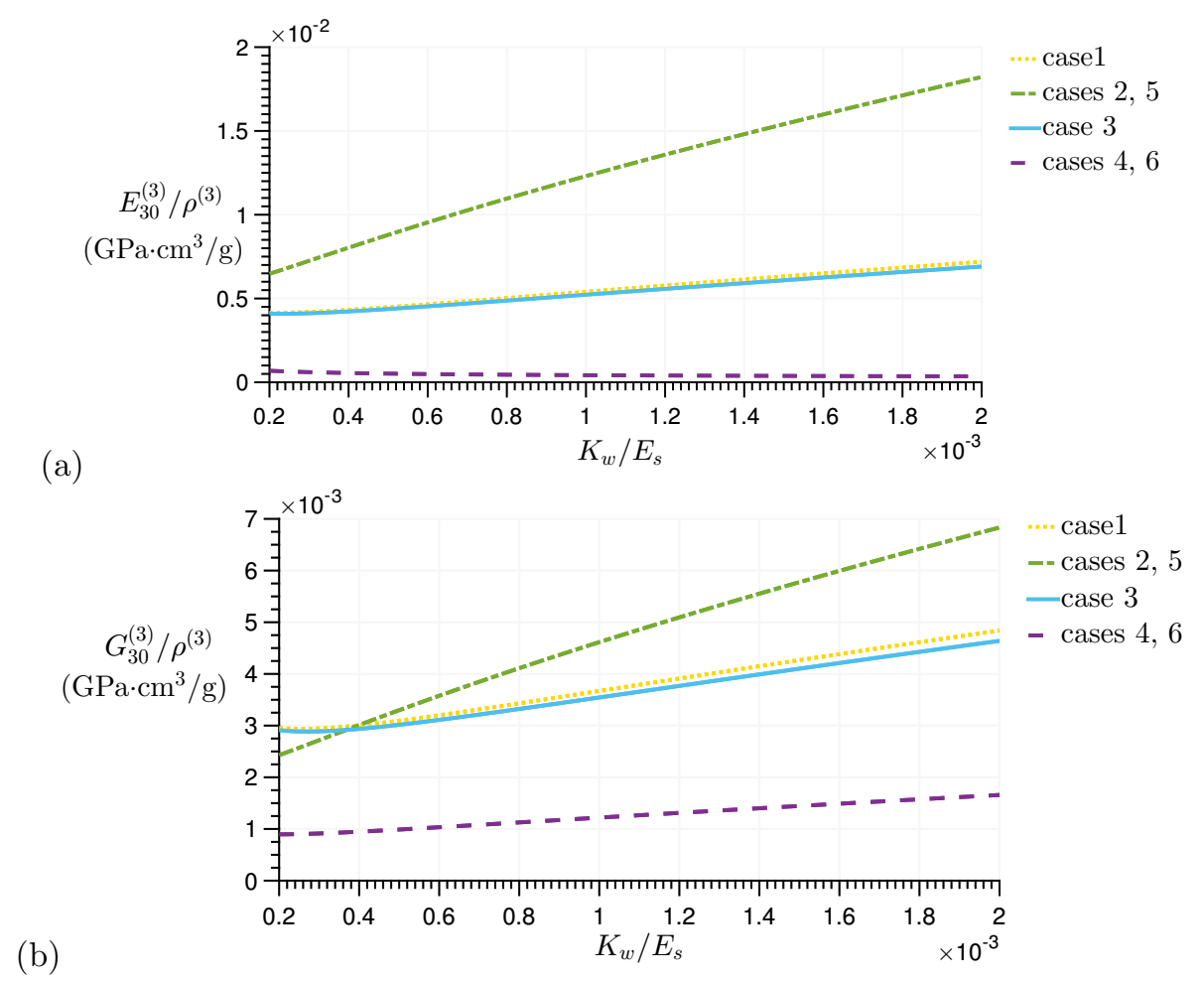

Figure 10: The influence of $K_{w}$ in the stiffness-to-density ratio associated with the axis rotated counterclockwise by $30^{\circ}$ from $\left(\mathbf{e}_{1}, \mathbf{e}_{2}\right)$, with $\lambda=0.02$ : (a) Young's modulus, (b) Shear modulus.

Table 5: Hierarchical configurations with different cell topologies.

\begin{tabular}{cccc}
\hline & level 1 & level 2 & level 3 \\
\hline case 1 & square & hexagonal & triangular \\
case 2 & square & triangular & hexagonal \\
case 3 & hexagonal & square & triangular \\
case 4 & hexagonal & triangular & square \\
case 5 & triangular & square & hexagonal \\
case 6 & triangular & hexagonal & square \\
\hline
\end{tabular}




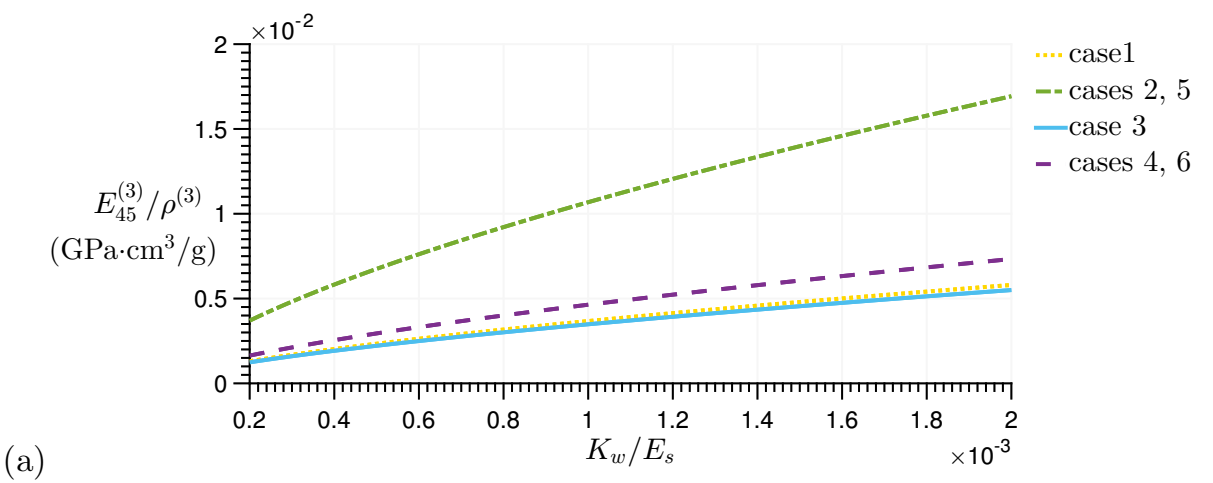

(a)

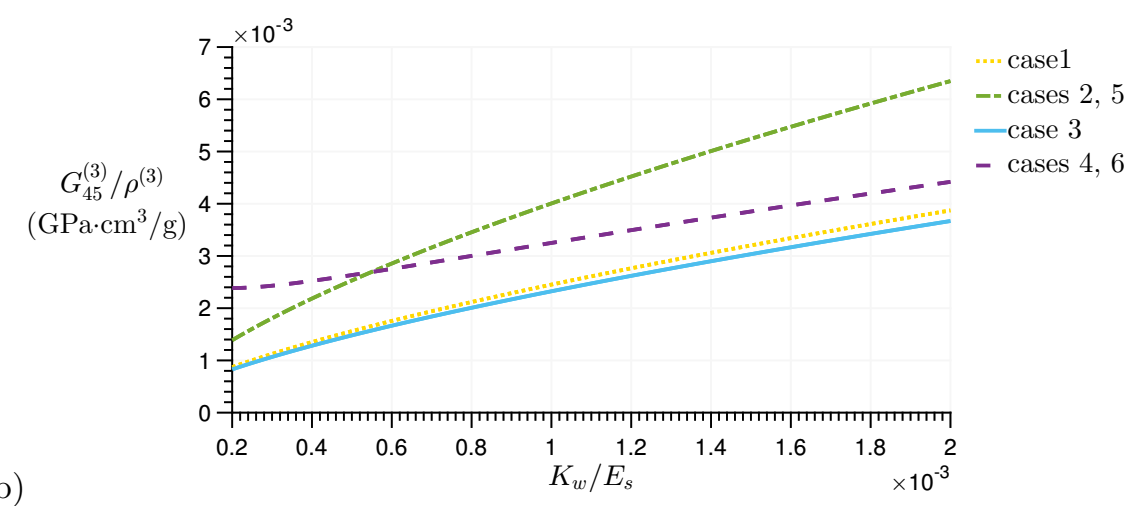

Figure 11: The influence of $K_{w}$ in the stiffness-to-density ratio associated with the axis rotated counterclockwise by $45^{\circ}$ from $\left(\mathbf{e}_{1}, \mathbf{e}_{2}\right)$, with $\lambda=0.02$ : (a) Young's modulus, (b) Shear modulus. 


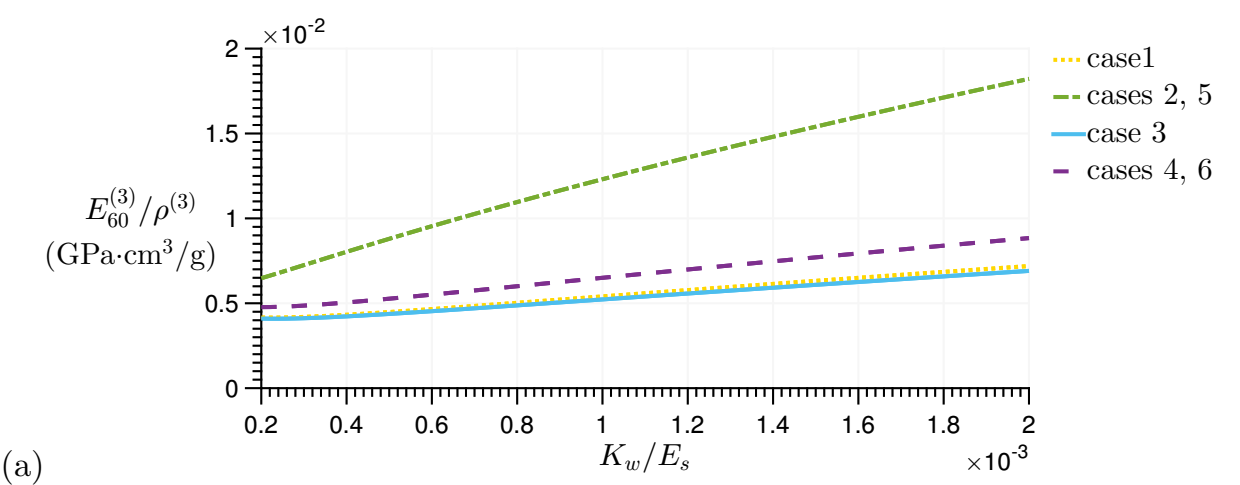

(a)

(b)

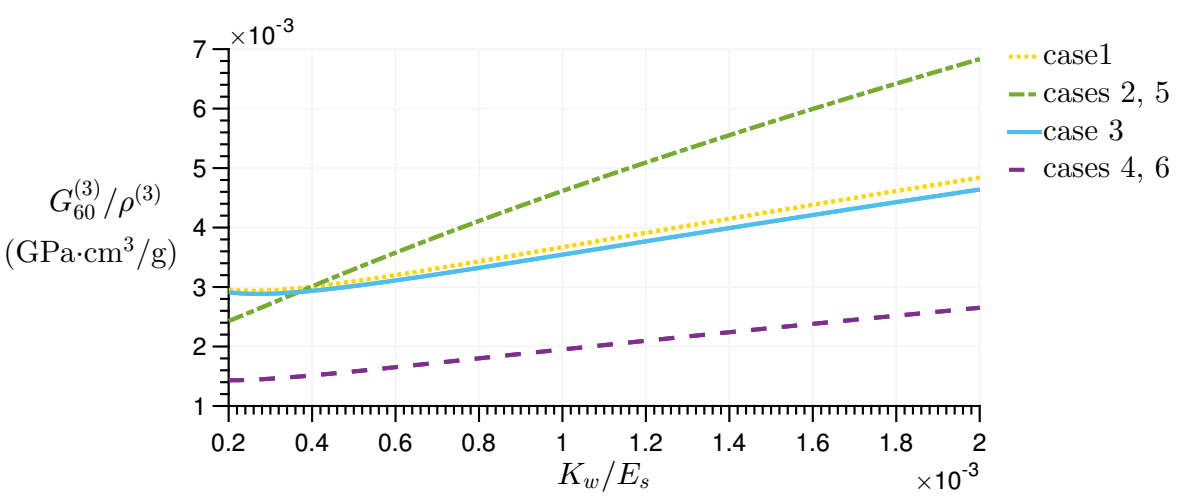

Figure 12: The influence of $K_{w}$ in the stiffness-to-density ratio associated with the axis rotated counterclockwise by $60^{\circ}$ from $\left(\mathbf{e}_{1}, \mathbf{e}_{2}\right)$, with $\lambda=0.02$ : (a) Young's modulus, (b) Shear modulus. 


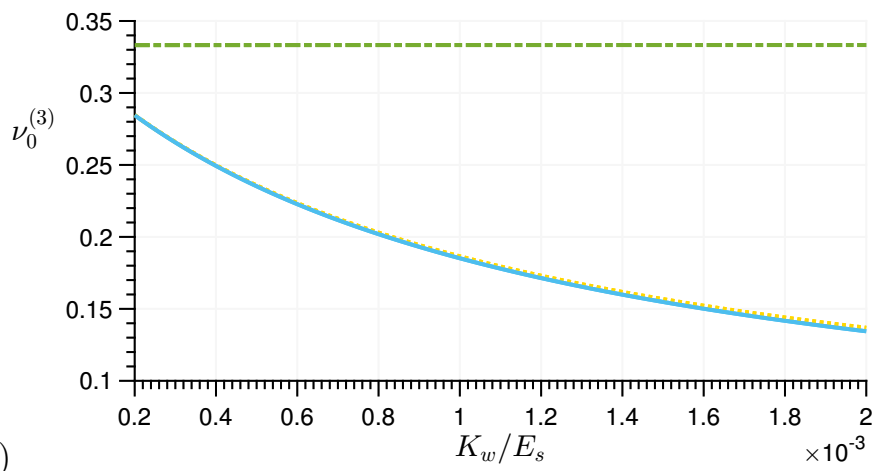

... case 1

- - cases 2,5

- case 3

(a)

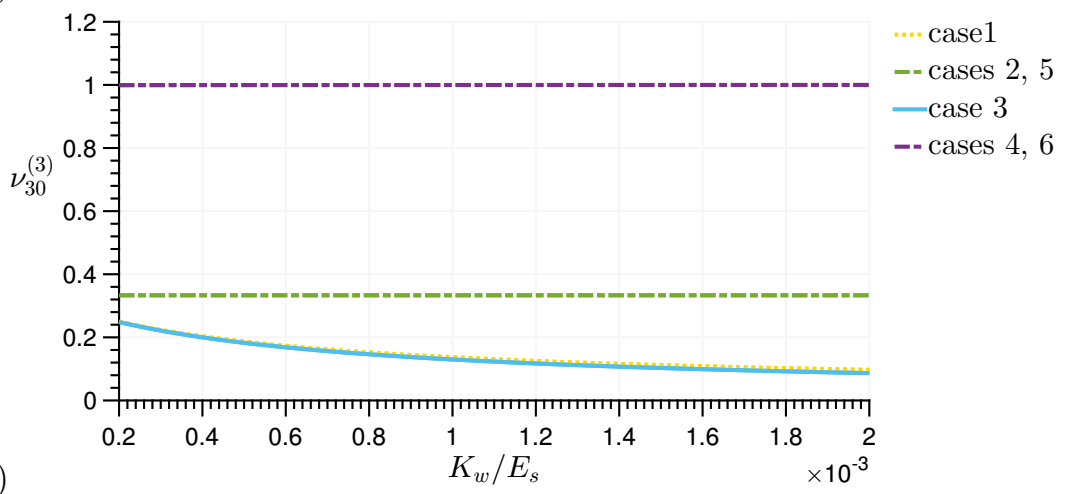

(b)

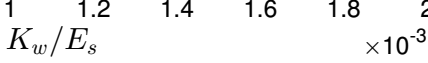

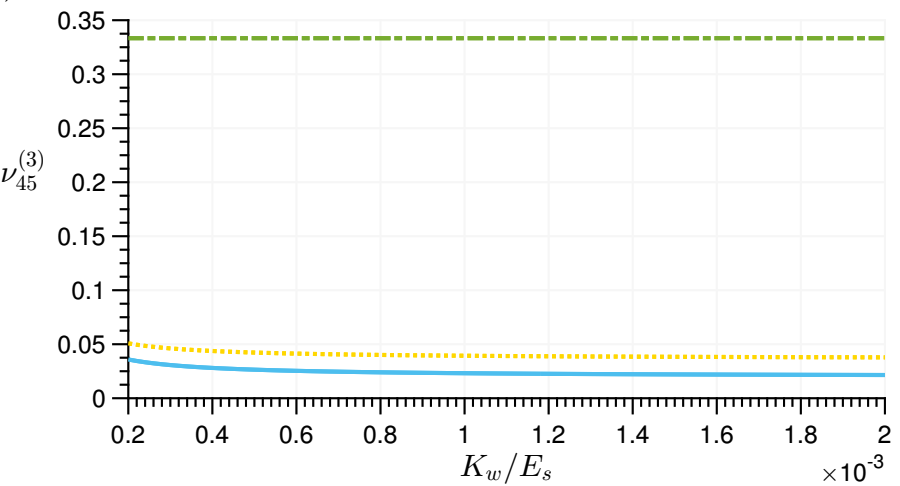

-- cases 2,5

- case 3

(c)

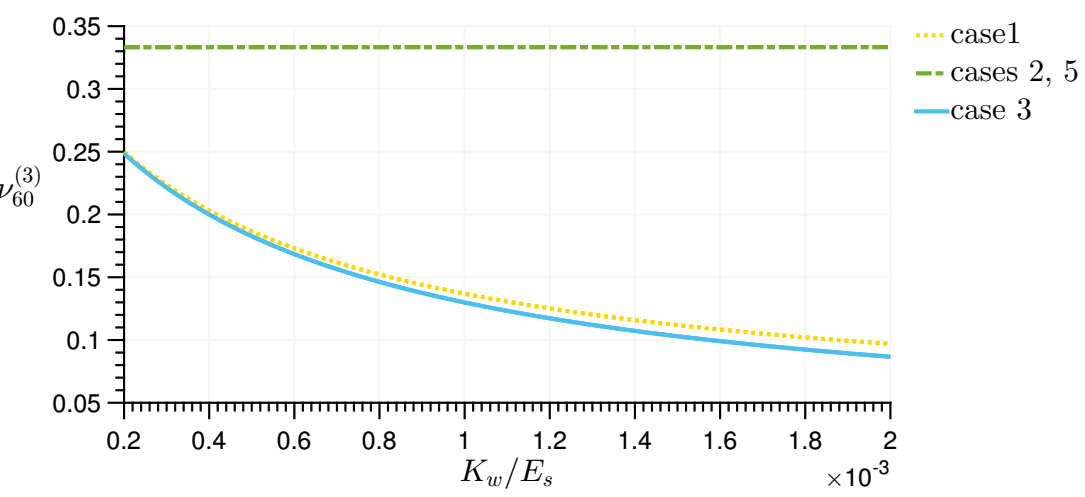

24

Figure 13: The influence of $K_{w}$ in the Poisson's ratio associated with (a) the axis $\left(\mathbf{e}_{1}, \mathbf{e}_{2}\right)$ and with the axis rotated counterclockwise from $\left(\mathbf{e}_{1}, \mathbf{e}_{2}\right)$ by (b) $30^{\circ}$, (c) $45^{\circ}$, (d) $60^{\circ}$, in the case of $\lambda=0.02$. 
The in-plane elastic properties of hierarchical composite cellular materials: synergy of hierarchy, material heterogeneity and cell topology at different levels - Appendices

\section{Appendix A. Relation between the Young's modulus of the filling material and the corresponding Winkler foundation constant}

As stated in Section 2, the Euler-Bernoulli beam on Winkler foundation model simulates the composite hexagonal microstructure at all levels. Specifically, a sequence of closely spaced independent linear-elastic springs approximates the cells filling material. Note that representing the material within the cells by a Winkler foundation is a simplification to obtain a more mathematically tractable problem. However, notwithstanding the limitations introduced, the analysis in [1] reveals the validity of the modeling approach based on the Winkler model. A suitable relation between the Young's modulus of the filling material, $E_{f}^{(i)}$ and the Winkler foundation constant, $k_{w}^{(i)}$, is also provided [1]:

$$
E_{f}^{(i)}=\frac{5 \sqrt{3}}{8} K_{w}^{(i)}, \quad i=1,2,3,
$$

been $K_{w}^{(i)}=k_{w}^{(i)} \ell^{(i)}$ and $\ell^{(i)}$ the length of the cell walls.

Assuming that the material inside the cells is a honeycomb made of an aluminum alloy as the whole hierarchical composite cellular structure analyzed in the present paper, leads to $[2]$

$$
\begin{gathered}
\frac{\rho_{f}^{(i)}}{\rho_{s}}=\frac{2}{\sqrt{3}} \lambda_{f}^{(i)}, \quad i=1,2,3 \\
\frac{E_{f}^{(i)}}{E_{s}}=\frac{4}{\sqrt{3}}\left(\lambda_{f}^{(i)}\right)^{3}, \quad i=1,2,3
\end{gathered}
$$

with $\rho_{f}^{(i)}, E_{f}^{(i)}$ and $\rho_{s}, E_{s}$, respectively, the density and the Young's modulus of the honeycomb and of the constituent material. Also, $\lambda_{f}^{(i)}$ is the ratio between the thickness and the length of the cell arms. From (A.2)

$$
\lambda_{f}^{(i)}=\frac{\sqrt{3}}{2}\left(\frac{\rho_{f}^{(i)}}{\rho_{s}}\right), \quad i=1,2,3 .
$$

Substituting (A.4) into (A.3) gives, in view of (A.1),

$$
K_{w}^{(i)}=\frac{4 \sqrt{3}}{5} E_{s}\left(\frac{\rho_{f}^{(i)}}{\rho_{s}}\right)^{3}, \quad i=1,2,3 .
$$


Finally, from the self-similar condition

$$
\rho_{f}^{(i)}=\rho_{f}=\alpha \rho_{s} \quad i=1,2,3
$$

and the assumption

$$
\lambda_{f}^{(i)}=\lambda_{f}, \quad i=1,2,3,
$$

follows

$$
K_{w}^{(i)}=K_{w}=\frac{4 \sqrt{3}}{5} \alpha^{3} E_{s}, \quad i=1,2,3 .
$$

In particular, assuming $\alpha=0.4,0.2,0.1,0.05$, (A.8) provides, on order, $K_{w}=10^{-1} E_{s}$, $10^{-2} E_{s}, 10^{-3} E_{s}, 10^{-4} E_{s}$.

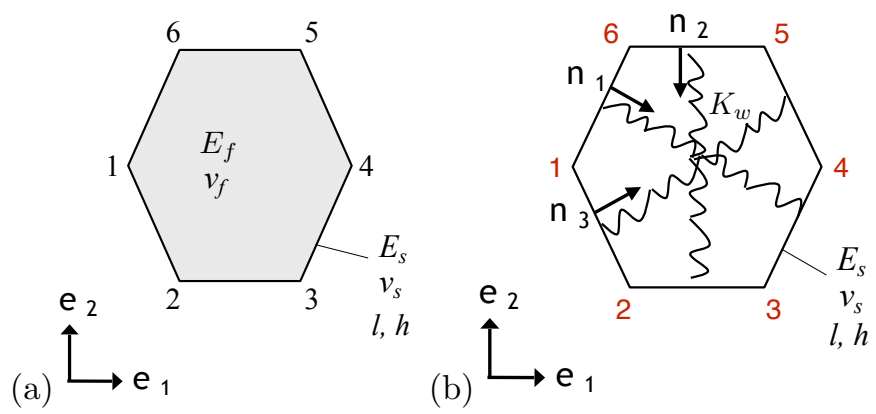

Figure A.1: Equivalence between the elastic moduli of the filling material and corresponding spring. (a) Filling material as a classical continuum, (b) Filling material as a Winkler foundation.

\section{Appendix B. A continuum model for composite cellular material with square microstructure}

Appendix B.1. Elastic energy

As Figure (B.2a) shows, a sequence of elastic beams of length $\ell$ forming a periodic array of square cells reproduces a cellular composite material with a square microstructure. Also, an elastic foundation represents the elastic material filling the cells. In particular, the Winkler foundation model simulates each beam, as in [1]. The unit cell of the periodic array (Fig. (B.2b)) is composed by the central node (0) and the four external nodes (1), (2), (3), (4), linked by the elastic beams (0)-(1), (0)-(2), (0)-(3), (0)-(4), represented by the vectors

$$
\mathbf{b}_{1}=(\ell, 0), \quad \mathbf{b}_{2}=(0, \ell), \quad \mathbf{b}_{3}=-\mathbf{b}_{1}, \quad \mathbf{b}_{4}=-\mathbf{b}_{2} .
$$

Finally, the area of the unit cell is $A_{0}=\ell^{2}$.

The analysis of the representative cell of the microstructure provides, firstly, the strain energy density of the discrete structure. Its continuum approximation is the consequence of particular assumptions. 
(a)

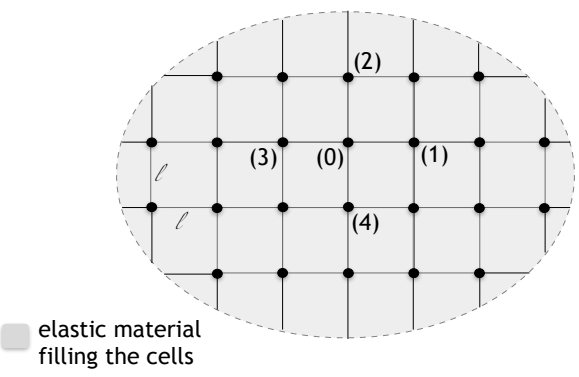

(b)

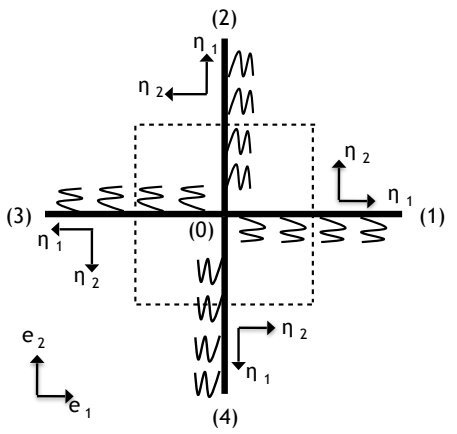

(c) L L ${ }_{1}$

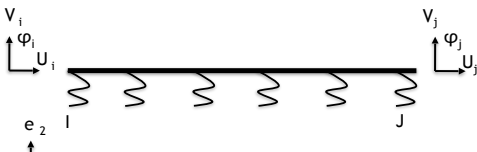

Figure B.2: (a) The square microstructure, (b) The unit cell, (c) The beam on Winkler elastic foundation.

First of all, the elastic energy of each beam

$$
w^{e}=\frac{1}{2}\left(\mathbf{u}^{e}\right)^{T} \cdot \mathbf{k}_{b}^{e} \mathbf{u}^{e}+\frac{1}{2}\left(\frac{1}{2}\left(\boldsymbol{\Delta} \mathbf{u}^{e, a}\right)^{T} \cdot \mathbf{k}_{w f}^{e} \boldsymbol{\Delta} \mathbf{u}^{e, a}\right)+\frac{1}{2}\left(\frac{1}{2}\left(\boldsymbol{\Delta} \mathbf{u}^{e, b}\right)^{T} \cdot \mathbf{k}_{w f}^{e} \boldsymbol{\Delta} \mathbf{u}^{e, b}\right)
$$

derives by superposition principle due to the assumption of linear elastic beam. In particular, $\mathbf{u}^{e}=\left[\mathbf{u}_{i}, \mathbf{u}_{j}\right]^{T}=\left[u_{i}, v_{i}, \varphi_{i}, u_{j}, v_{j}, \varphi_{j}\right]^{T}$ is the generalized vector of nodal displacement expressed in the local reference and

$$
\begin{gathered}
\Delta \mathbf{u}^{e, a}=\left[\boldsymbol{\Delta} \mathbf{u}_{i}^{a}, \boldsymbol{\Delta} \mathbf{u}_{j}^{a}\right]^{T}=\left[\Delta u_{i}^{a}, \Delta v_{i}^{a}, \Delta \varphi_{i}^{a}, \Delta u_{j}^{a}, \Delta v_{j}^{a}, \Delta \varphi_{j}^{a}\right]^{T}, \\
\Delta \mathbf{u}^{e, b}=\left[\boldsymbol{\Delta} \mathbf{u}_{i}^{b}, \boldsymbol{\Delta} \mathbf{u}_{j}^{b}\right]^{T}=\left[\Delta u_{i}^{b}, \Delta v_{i}^{b}, \Delta \varphi_{i}^{b}, \Delta u_{j}^{b}, \Delta v_{j}^{b}, \Delta \varphi_{j}^{b}\right]^{T}
\end{gathered}
$$

is the elongation of the springs a, the first, and of the springs b, the second (Fig. (B.3)). Note that the factor $1 / 2$ in the second and third term of (B.2), is due to the fact that the springs are shared by two opposite beams and contribute only half of its strain energy to the unit cell. See Appendix D for further details.

The terms $\mathbf{k}_{b}^{e}$ and $\mathbf{k}_{w f}^{e}$ in (B.2) are, respectively, the stiffness matrix of the classical elastic beam and of the Winkler foundation [3], denoted by lowercase letters since they are expressed in the local reference (see [1] for a detailed description). Their components are

$$
\mathbf{k}_{b}^{e}=\left[\begin{array}{cccccc}
C_{\ell} / \ell & 0 & 0 & -C_{\ell} / \ell & 0 & 0 \\
0 & 12 D_{\ell} / \ell^{3} & 6 D_{\ell} / \ell^{2} & 0 & -12 D_{\ell} / \ell^{3} & 6 D_{\ell} / \ell^{2} \\
0 & 6 D_{\ell} / \ell^{2} & 4 D_{\ell} / \ell & 0 & -6 D_{\ell} / \ell^{2} & 2 D_{\ell} / \ell \\
-C_{\ell} / \ell & 0 & 0 & C_{\ell} / \ell & 0 & 0 \\
0 & -12 D_{\ell} / \ell^{3} & -6 D_{\ell} / \ell^{2} & 0 & 12 D_{\ell} / \ell^{3} & -6 D_{\ell} / \ell^{2} \\
0 & 6 D_{\ell} / \ell^{2} & 2 D_{\ell} / \ell & 0 & -6 D_{\ell} / \ell^{2} & 4 D_{\ell} / \ell
\end{array}\right]
$$



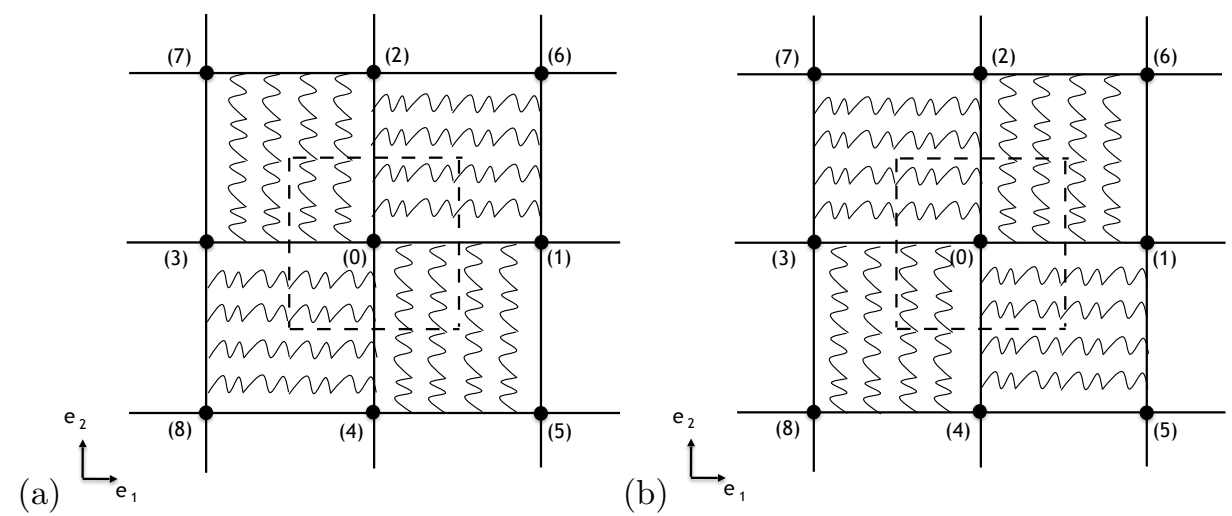

Figure B.3: Square microstructure: (a) Springs a, (b) Springs $b$.

and

$$
\mathbf{k}_{w f}^{e}=\left[\begin{array}{cccccc}
0 & 0 & 0 & 0 & 0 & 0 \\
0 & 3 K_{w} / 35 & 11 K_{w} \ell / 210 & 0 & 9 K_{w} / 70 & -13 K_{w} \ell / 420 \\
0 & 11 K_{w} \ell / 210 & K_{w} \ell^{2} / 105 & 0 & 13 K_{w} \ell / 420 & -K_{w} \ell^{2} / 140 \\
0 & 0 & 0 & 0 & 0 & 0 \\
0 & 9 K_{w} / 70 & 13 K_{w} \ell / 420 & 0 & 13 K_{w} / 35 & -11 K_{w} \ell / 210 \\
0 & -3 K_{w} \ell / 420 & -K_{w} \ell^{2} / 140 & 0 & -11 K_{w} \ell / 210 & K_{w} \ell^{2} / 105
\end{array}\right],
$$

with $K_{w}=k_{w} \ell, k_{w}$ the Winkler foundation constant per unit width, $C_{\ell}=\frac{E_{s} h}{1-\nu_{s}^{2}}$ and $D_{\ell}=\frac{E_{s} h^{3}}{12\left(1-\nu_{s}^{2}\right)}$, respectively, the tensile and bending stiffness (per unit width) of the beams, $h$ the thickness of the arms, $E_{s}$ and $\nu_{s}$ the Young's modulus and the Poisson's ratio of the cell walls material.

As it can be seen, the elastic energy is the sum of three terms. The first one,

$$
\frac{1}{2}\left(\mathbf{u}^{e}\right)^{T} \cdot \mathbf{k}_{b}^{e} \mathbf{u}^{e},
$$

corresponding to the classical elastic beam, while the second and the third,

$$
\frac{1}{2}\left(\frac{1}{2}\left(\boldsymbol{\Delta} \mathbf{u}^{e, a}\right)^{T} \cdot \mathbf{k}_{w f}^{e} \boldsymbol{\Delta} \mathbf{u}^{e, a}\right), \quad \frac{1}{2}\left(\frac{1}{2}\left(\boldsymbol{\Delta} \mathbf{u}^{e, b}\right)^{T} \cdot \mathbf{k}_{w f}^{e} \boldsymbol{\Delta} \mathbf{u}^{e, b}\right),
$$

related to the Winkler foundation and, in particular, to the elongation of the springs a, the first, and of the springs b, the second (Fig. (B.3)).

The elastic energy of the unit cell, $W$, derives from that of the four beams it consists of. In particular, expressing (B.2) in the global reference and summing the elastic energies of the four beams, leads to

$$
W=\frac{W^{1}+W^{2}+W^{3}+W^{4}}{2} .
$$

The presence of the factor 2 in (B.9) is related to the fact that each beam is shared by two adjacent cells. So, each member contributes only half of its strain energy to the representative cell. 
The assumption that in the limit $\ell \rightarrow 0$ there exist the continuous displacement and microrotation fields $\hat{\mathbf{u}}(\cdot)$ and $\hat{\varphi}(\cdot)$, and that the discrete variables previously introduced to represent the degrees of freedom (displacements and rotations) of the external nodes of the unit cell can be expressed by [4]:

$$
\mathbf{u}_{j}=\hat{\mathbf{u}}_{0}+\nabla \hat{\mathbf{u}} \mathbf{b}_{j}+\frac{1}{2} \nabla^{2} \hat{\mathbf{u}} \mathbf{b}_{j}^{2}, \quad \varphi_{j}=\hat{\varphi}_{0}+\nabla \hat{\varphi} \mathbf{b}_{j}+\frac{1}{2} \nabla^{2} \hat{\varphi} \mathbf{b}_{j}^{2}, \quad j=1,2,3,4
$$

provides the continuum description of the discrete structure. In (B.10), $\mathbf{b}_{j}$ are the vectors formerly defined, $\hat{\mathbf{u}}_{\mathbf{0}}$ and $\hat{\varphi}_{0}$ are the values of $\hat{\mathbf{u}}(\cdot)$ and $\hat{\varphi}(\cdot)$ at the central point of the cell in the continuum description. The substitution of (B.10) into (B.9) gives the strain energy of the unit cell as a function of the fields $\hat{\mathbf{u}}(\cdot)$ and $\hat{\varphi}(\cdot)$. Finally, dividing the expression that turns out by the area of the unit cell, $A_{0}$, leads to the strain energy density in the continuum approximation

$$
\begin{gathered}
w=\frac{C_{\ell} \ell^{2}\left(\varepsilon_{11}^{2}+\varepsilon_{22}^{2}\right)+24 D_{\ell}\left(\varepsilon_{12}^{2}+(\omega-\hat{\varphi})^{2}\right)-2 D_{\ell} \ell^{2}\left(\hat{\varphi}_{, 1}^{2}+\hat{\varphi}_{, 2}^{2}\right)-12 D_{\ell} \ell \varepsilon_{12} \hat{\varphi}_{, 2}}{2 \ell^{3}}+ \\
\frac{K_{w}\left(210\left(\varepsilon_{11}^{2}+\varepsilon_{22}^{2}\right)+\ell^{2}\left(\hat{\varphi}_{, 1}^{2}+\hat{\varphi}_{, 2}^{2}\right)\right)}{420},
\end{gathered}
$$

been $\varepsilon_{\alpha \beta}=\frac{1}{2}\left(\hat{u}_{\alpha, \beta}+\hat{u}_{\beta, \alpha}\right)$ the infinitesimal strains, $\omega=\frac{1}{2}\left(\hat{u}_{1,2}-\hat{u}_{2,1}\right)$ the infinitesimal rotation, $\hat{\varphi}_{, \alpha}$ the microrotation gradients. Note that in (B.11) only the first order derivatives are retained, except for the terms of the form $\hat{\varphi} \hat{\varphi}, \alpha \alpha$ that can be integrated by parts and result in first order derivative terms. Retaining this terms, in particular, is important to maintain joint equilibrium, as pointed out in [4], [5], [6].

Moreover, after rewriting (B.11) in terms of $c \equiv C_{\ell} / \ell=\frac{E_{s}(h / \ell)}{1-\nu_{s}^{2}}$ and $d \equiv D_{\ell} / \ell^{3}=$ $\frac{E_{s}(h / \ell)^{3}}{12\left(1-\nu_{s}^{2}\right)}$, it emerges that in the resulting energy the coefficients scale with different order in $\ell$, as in [7], [1]. Specifically, the microrotation gradients scale with first order in $\ell$, while the others coefficients are independent of $\ell$. Accordingly, in the limit $\ell \rightarrow 0$ the contribution of the microrotation gradients is missing and, as in [1], the equivalent continuum is non-polar. Consequently, the strain energy density in the continuum description is

$$
w=\frac{c\left(\varepsilon_{11}^{2}+\varepsilon_{22}^{2}\right)+24 d\left(\varepsilon_{12}^{2}+(\omega-\hat{\varphi})^{2}\right)}{2 \ell^{3}}+\frac{K_{w}\left(210\left(\varepsilon_{11}^{2}+\varepsilon_{22}^{2}\right)\right.}{420} .
$$

Appendix B.2. Constitutive equations

The constitutive equations

$$
\sigma=\frac{1}{A_{0}} \frac{\partial W}{\partial \nabla \hat{\mathbf{u}}}
$$

with $\sigma$ the Cauchy-type stress tensor, follows from (B.12). 
In particular, it emerges that $\sigma$ is a non-symmetric tensor and its components are

$$
\begin{aligned}
& \sigma_{11}=\sigma_{11}^{s y m}=\left(\frac{C_{\ell}}{\ell}+K_{w}\right) \varepsilon_{11}, \\
& \sigma_{22}=\sigma_{22}^{s y m}=\left(\frac{C_{\ell}}{\ell}+K_{w}\right) \varepsilon_{22}, \\
& \sigma_{12}^{s y m}=\sigma_{21}^{s y m}=\frac{12 D_{\ell}}{\ell^{3}} \varepsilon_{12}, \\
& \sigma_{12}^{s k w}=-\sigma_{21}^{s k w}=\frac{12 D_{\ell}}{\ell^{3}}(\omega-\hat{\varphi}), \\
& \sigma_{12}=\sigma_{12}^{s y m}+\sigma_{12}^{s k w}, \quad \sigma_{21}=\sigma_{21}^{s y m}+\sigma_{21}^{s k w},
\end{aligned}
$$

with $\sigma_{\gamma \delta}^{s y m}$ and $\sigma_{\gamma \delta}^{s k w}$, in turn, the symmetric and skew-symmetric parts of $\sigma$. See [1] for further details.

\section{Appendix B.3. Elastic constants}

Simple mathematical manipulations lead to the elastic constants in the continuum approximation. Specifically, the stress state $\sigma_{11} \neq 0, \sigma_{22}=\sigma_{12}=\sigma_{21}=0$ provide, in view of (B.14) and Hooke's law $\sigma_{11}^{\text {sym }}=E_{1}^{*} \varepsilon_{11}$, the Young's modulus in the $\mathbf{e}_{1}$ direction:

$$
E_{1}^{*}=\frac{\sigma_{11}}{\varepsilon_{11}}=\frac{E_{s} \lambda}{\left(1-\nu_{s}^{2}\right)}+K_{w}
$$

with $E_{s}$ and $\nu_{s}$, respectively, the Young's modulus and the Poisson's ratio of the cell walls material, $\lambda=h / \ell$ the ratio between the thickness and the length of the beams. The related Poisson's ratio $\nu_{12}^{*}=-\varepsilon_{22} / \varepsilon_{11}$ is

$$
\nu_{12}^{*}=0 .
$$

Similarly, the stress state defined as $\sigma_{22} \neq 0, \sigma_{11}=\sigma_{12}=\sigma_{21}=0$ gives the Young's modulus in the $\mathbf{e}_{\mathbf{2}}$ direction:

$$
E_{2}^{*}=\frac{\sigma_{22}}{\varepsilon_{22}}=\frac{E_{s} \lambda}{\left(1-\nu_{s}^{2}\right)}+K_{w}
$$

and the related Poisson's ratio $\nu_{21}^{*}=-\varepsilon_{11} / \varepsilon_{22}=0$.

As it can be seen, it emerges that $E_{1}^{*}=E_{2}^{*} \equiv E^{*}$ and $\nu_{12}^{*}=\nu_{21}^{*} \equiv \nu^{*}$, with $E^{*}$ and $\nu^{*}$ stands for the Young's modulus, the first, and the Poisson's ratio, the second, of the approximated continuum.

The tangential elastic modulus, $G^{*}=\sigma_{12}^{s y m} / 2 \varepsilon_{12}$, it is easily obtained by considering the stress state $\sigma_{12}^{\text {sym }} \neq 0, \sigma_{11}=\sigma_{22}=0$ :

$$
G^{*}=\frac{\sigma_{12}^{\text {sym }}}{2 \varepsilon_{12}}=\frac{E_{s} \lambda^{3}}{2\left(1-\nu_{s}^{2}\right)} .
$$


It should be noted that the elastic moduli derived do not satisfy the classical relation for isotropic materials, $G^{*}=\frac{E^{*}}{2\left(1+\nu^{*}\right)}$. The effective elastic constants are equal only in the $\mathbf{e}_{1}$ and $\mathbf{e}_{2}$ direction [2], [8].

Analogous calculations, in conjunction with the classic transformation equations for stress and strain, provide the effective elastic moduli associated with different axis. In particular, denoting with $E_{\theta}^{*}, \nu_{\theta}^{*}, G_{\theta}^{*}$, respectively, the Young's modulus, Poisson's ratio and shear modulus associated with the axis rotated counterclockwise through an angle of $\theta$ from $\left(\mathbf{e}_{1}, \mathbf{e}_{2}\right)$, it emerges:

$$
\begin{gathered}
E_{\theta}^{*}=\frac{\lambda^{3} E_{s}\left(K_{w}\left(1-\nu_{s}^{2}\right)+\lambda E_{s}\right)}{\left(1-\nu_{s}^{2}\right)\left(c^{4} \lambda^{3} E_{s}+\lambda^{3} s^{4} E_{s}+2 c^{2} s^{2}\left(K_{w}\left(1-\nu_{s}^{2}\right)+\lambda E_{s}\right)\right)}, \\
\nu_{\theta}^{*}=\frac{2 c^{2} s^{2}\left(K_{w}\left(1-\nu_{s}^{2}\right)+\lambda E_{s}-\lambda^{3} E_{s}\right)}{c^{4} \lambda^{3} E_{s}+\lambda^{3} s^{4} E_{s}+2 c^{2} s^{2}\left(K_{w}\left(1-\nu_{s}^{2}\right)+\lambda E_{s}\right)}, \\
G_{\theta}^{*}=\frac{\lambda^{3} E_{s}\left(K_{w}\left(1-\nu_{s}^{2}\right)+\lambda E_{s}\right)}{4\left(1-\nu_{s}^{2}\right)\left(\left(c^{2}-s^{2}\right)^{2} K_{w}\left(1-\nu_{s}^{2}\right)+\lambda\left(c^{4}+2 c^{2}\left(-1+2 \lambda^{2}\right) s^{2}+s^{4}\right) E_{s}\right)} .
\end{gathered}
$$

To simplify the notation, $c$ and $s$ stand, respectively, for $\cos \theta$ and $\sin \theta$.

Appendix B.4. Comparison between the analytical and numerical approach

Writing the constitutive equations derived in Section B.2 in a compact way, provide

$$
\left[\begin{array}{l}
\sigma_{11}^{s y m} \\
\sigma_{22}^{s y m} \\
\sigma_{12}^{s y m}
\end{array}\right]=\left[\begin{array}{lll}
C_{11} & C_{12} & C_{13} \\
C_{21} & C_{22} & C_{23} \\
C_{31} & C_{32} & C_{33}
\end{array}\right]\left[\begin{array}{l}
\varepsilon_{11} \\
\varepsilon_{22} \\
\varepsilon_{12}
\end{array}\right],
$$

been

$$
\begin{gathered}
C_{11}=C_{22}=\frac{C_{\ell}}{\ell}+K_{w}=\frac{E_{s} \lambda}{\left(1-\nu_{s}^{2}\right)}+K_{w}, \\
C_{33} \quad=\frac{12 D_{\ell}}{\ell^{3}}=\frac{E_{s} \lambda^{3}}{\left(1-\nu_{s}^{2}\right)} \\
C_{12}=C_{21}=C_{13}=C_{23}=C_{31}=C_{32}=0 .
\end{gathered}
$$

In terms of stress,

$$
\left[\begin{array}{l}
\varepsilon_{11} \\
\varepsilon_{22} \\
\varepsilon_{12}
\end{array}\right]=\left[\begin{array}{lll}
C_{11}^{*} & C_{12}^{*} & C_{13}^{*} \\
C_{21}^{*} & C_{22}^{*} & C_{23}^{*} \\
C_{31}^{*} & C_{32}^{*} & C_{33}^{*}
\end{array}\right]\left[\begin{array}{l}
\sigma_{11}^{\text {sym }} \\
\sigma_{22}^{\text {sym }} \\
\sigma_{12}^{\text {sym }}
\end{array}\right]
$$

where

$$
\begin{gathered}
C_{11}^{*}=C_{22}^{*} \quad=\frac{C_{22} C_{33}}{C_{22}^{2} C_{33}-C_{12}^{2} C_{33}}=\frac{\left(1-\nu_{s}^{2}\right)}{K_{w}\left(1-\nu_{s}^{2}\right)+E_{s} \lambda}, \\
C_{33}^{*} \quad=\frac{C_{22}^{2}-C_{12}^{2}}{C_{22}^{2} C_{33}-C_{12}^{2} C_{33}}=\frac{\left(1-\nu_{s}^{2}\right)}{E_{s} \lambda^{3}}, \\
C_{12}^{*}=C_{21}^{*}=C_{13}^{*}=C_{23}^{*}=C_{31}^{*}=C_{32}^{*}=0 .
\end{gathered}
$$


Finite element simulations on a computational model of the microstructure evaluate the accuracy of the theoretical model. In particular, the Euler-Bernoulli beam on Winkler foundation elements model the composite square microstructure. The cell wall material, isotropic linear elastic for assumption, has Young's modulus $E_{s}=79 \mathrm{GPa}$, Poisson's ratio $\nu_{s}=0.35$ and thickness $h=0.1 \ell$. In terms of Winkler foundation, $K_{w}=10^{-2} E_{s}$. The numerical analysis involve a $50 \times 50 \mathrm{~mm}$ square domain discretized in an increasing number of square cells of gradually smaller length $\ell$. As done in [1], the load conditions are the uniaxial compression, uniaxial traction and in-plane shear. Specifically, forces of the same intensity acting at the boundary, unconstrained nodes of the domain simulate the loading states. The corresponding effective stiffness components are derived as the ratio between the average volume strain,

$$
\bar{\varepsilon}_{i j}=\frac{1}{V} \int_{V} \varepsilon_{i j} d V, \quad i, j=1,2,
$$

and the applied stress. Referring the interested reader to [1] for a comprehensive description, in the case of forces acting horizontally, (B.24) takes the form

$$
\bar{\varepsilon}^{(1)}=\left[\begin{array}{c}
\bar{\varepsilon}_{11}^{(1)} \\
\bar{\varepsilon}_{22}^{(1)} \\
\bar{\varepsilon}_{12}^{(1)}
\end{array}\right]=\left[\begin{array}{lll}
C_{11}^{*} & C_{12}^{*} & C_{13}^{*} \\
C_{21}^{*} & C_{22}^{*} & C_{23}^{*} \\
C_{31}^{*} & C_{32}^{*} & C_{33}^{*}
\end{array}\right]\left[\begin{array}{c}
\sigma_{11} \\
0 \\
0
\end{array}\right]=\left[\begin{array}{c}
C_{11}^{*} \sigma_{11} \\
C_{21}^{*} \sigma_{11} \\
C_{31}^{*} \sigma_{11}
\end{array}\right]
$$

been $\sigma_{11}$ the applied stress, $\bar{\varepsilon}^{(1)}$ the corresponding strain vector,

$$
\bar{\varepsilon}_{i j}^{(1)}=\frac{1}{V} \int_{V} \varepsilon_{i j}^{(1)} d V, \quad i, j=1,2,
$$

and $V$ is the volume of the domain. Accordingly,

$$
C_{11}^{*}=\frac{\bar{\varepsilon}_{11}^{(1)}}{\sigma_{11}}, \quad C_{21}^{*}=\frac{\bar{\varepsilon}_{22}^{(1)}}{\sigma_{11}}, \quad C_{31}^{*}=\frac{\bar{\varepsilon}_{12}^{(1)}}{\sigma_{11}},
$$

Note that the present analysis involve a domain with unitary width, composed by a sequence of discrete beams having the same length $\ell$ and the same thickness $h$. Consequently, denoting by $s$ the parametric coordinate along the length of the beam $(0 \leq s \leq \ell)$ and remembering that

$$
\begin{gathered}
\varepsilon_{i j}(s)=\frac{1}{2}\left(\frac{\partial u_{i}(s)}{\partial x_{j}}+\frac{\partial u_{j}(s)}{\partial x_{i}}\right), \\
\bar{\varepsilon}_{i j}^{(1)}=\frac{\sum_{m=1}^{n_{b}} \frac{1}{2}\left(\left(u_{i}(\ell)-u_{i}(0)\right) \frac{\partial s}{\partial x_{j}}+\left(u_{j}(\ell)-u_{j}(0)\right) \frac{\partial s}{\partial x_{i}}\right)_{m}}{n_{b} \ell} .
\end{gathered}
$$

been $n_{b}$ the number of the beams. Furthermore, the classical continuum mechanics provides the Young's modulus, $E_{1}^{*}$, and the related Poisson's ratio $\nu_{12}^{*}$ :

$$
E_{1}^{*}=\frac{\sigma_{11}}{\bar{\varepsilon}_{11}^{(1)}}, \quad \nu_{12}^{*}=-\frac{\bar{\varepsilon}_{22}^{(1)}}{\bar{\varepsilon}_{11}^{(1)}} .
$$


Similarly, when the forces act vertically,

$$
\bar{\varepsilon}^{(2)}=\left[\begin{array}{c}
\bar{\varepsilon}_{11}^{(2)} \\
\bar{\varepsilon}_{22}^{(2)} \\
\bar{\varepsilon}_{12}^{(2)}
\end{array}\right]=\left[\begin{array}{lll}
C_{11}^{*} & C_{12}^{*} & C_{13}^{*} \\
C_{21}^{*} & C_{22}^{*} & C_{23}^{*} \\
C_{31}^{*} & C_{32}^{*} & C_{33}^{*}
\end{array}\right]\left[\begin{array}{c}
0 \\
\sigma_{22} \\
0
\end{array}\right]=\left[\begin{array}{c}
C_{12}^{*} \sigma_{22} \\
C_{22}^{*} \sigma_{22} \\
C_{32}^{*} \sigma_{22}
\end{array}\right],
$$

and, consequently,

$$
C_{12}^{*}=\frac{\bar{\varepsilon}_{11}^{(2)}}{\sigma_{22}}, \quad C_{22}^{*}=\frac{\bar{\varepsilon}_{22}^{(2)}}{\sigma_{22}}, \quad C_{32}^{*}=\frac{\bar{\varepsilon}_{12}^{(2)}}{\sigma_{22}} .
$$

Also,

$$
E_{2}^{*}=\frac{\sigma_{22}}{\bar{\varepsilon}_{22}^{(2)}}, \quad \nu_{21}^{*}=-\frac{\bar{\varepsilon}_{11}^{(2)}}{\bar{\varepsilon}_{22}^{(2)}} .
$$

with $\sigma_{22}$ the applied stress, $\bar{\varepsilon}^{(2)}$ the corresponding strain vector and $\bar{\varepsilon}_{i j}^{(2)}$ the average volume strain given by (B.26).

Lastly, the shear loading condition provides

$$
\begin{gathered}
\bar{\varepsilon}^{(3)}=\left[\begin{array}{c}
\bar{\varepsilon}_{11}^{(3)} \\
\bar{\varepsilon}_{22}^{(3)} \\
\bar{\varepsilon}_{12}^{(3)}
\end{array}\right]=\left[\begin{array}{lll}
C_{11}^{*} & C_{12}^{*} & C_{13}^{*} \\
C_{21}^{*} & C_{22}^{*} & C_{23}^{*} \\
C_{31}^{*} & C_{32}^{*} & C_{33}^{*}
\end{array}\right]\left[\begin{array}{c}
0 \\
0 \\
\sigma_{12}
\end{array}\right]=\left[\begin{array}{c}
C_{13}^{*} \sigma_{12} \\
C_{23}^{*} \sigma_{12} \\
C_{33}^{*} \sigma_{12}
\end{array}\right], \\
C_{13}^{*}=\frac{\bar{\varepsilon}_{11}^{(3)}}{\sigma_{12}}, \quad C_{23}^{*}=\frac{\bar{\varepsilon}_{22}^{(3)}}{\sigma_{12}}, \quad C_{33}^{*}=\frac{\bar{\varepsilon}_{12}^{(3)}}{\sigma_{12}} .
\end{gathered}
$$

and

$$
G^{*}=\frac{\sigma_{12}}{2 \bar{\varepsilon}_{12}^{(3)}}
$$

As before, $\sigma_{12}$ and $\bar{\varepsilon}^{(3)}$ are, in turn, the applied stress and the corresponding strain vector, while $\bar{\varepsilon}_{i j}^{(3)}$ is the average volume strain defined in (B.26).

Tables (B.1) and (B.2) present the outcome of the present study. In Table (B.1), in particular, the comparison involves the theoretical and numerical $C_{i j}^{*}$ constants. In Table (B.2), the theoretical and numerical elastic moduli. Both Table (B.1) and Table (B.2) show that the analytical quantities are in accordance with the numerical results.

\section{Appendix C. A continuum model for composite cellular material with equi- lateral triangular microstructure}

Appendix C.1. Elastic energy

As in Appendix B, a sequence of Euler-Bernoulli beams on Winkler foundation elements model the triangular microstructure (Fig. (C.4). As Figure (C.4b) shows, the unit cell of the periodic configuration is composed by the central node $(0)$ and the six external nodes (1), (2), (3), (4), (5), (6), linked by the elastic beams (0)-(1), (0)-(2), (0)-(3), (0)-(4), (0)-(5), (0)-(6), represented by the vectors

$$
\begin{array}{ccc}
\mathbf{b}_{1}=(\ell, 0), & \mathbf{b}_{2}=(\ell / 2, \sqrt{3} \ell / 2), & \mathbf{b}_{3}=(-\ell / 2, \sqrt{3} \ell / 2), \\
\mathbf{b}_{4}=-\mathbf{b}_{1}, & \mathbf{b}_{5}=-\mathbf{b}_{2}, & \mathbf{b}_{6}=-\mathbf{b}_{3} .
\end{array}
$$


Table B.1: Square microstructure: comparison between the analytical and numerical approach, $C_{i j}^{*}$ constants

\begin{tabular}{cccccc}
\hline No. cells & $\ell(\mathrm{mm})$ & $C_{11}^{*}$ & $C_{22}^{*}$ & $C_{33}^{*}$ & $C_{12}^{*}=C_{21}^{*}=C_{13}^{*}=C_{23}^{*}=C_{31}^{*}=C_{32}^{*}$ \\
\hline 10x10 & 5 & 0.08 & 0.10 & 9.39 & 0 \\
$50 \times 50$ & 1 & 0.10 & 0.11 & 10.70 & 0 \\
100x100 & 0.5 & 0.11 & 0.11 & 10.70 & 0 \\
$200 \times 200$ & 0.25 & 0.11 & 0.11 & 10.74 & 0 \\
$250 \times 250$ & 0.2 & 0.11 & 0.11 & 10.79 & 0 \\
$400 \times 400$ & 0.125 & 0.11 & 0.11 & 10.82 & 0 \\
$500 \times 500$ & 0.1 & 0.11 & 0.11 & 10.95 & 0 \\
Analytical results & & 0.11 & 0.11 & 11.11 & 0 \\
\hline
\end{tabular}

Table B.2: Square microstructure: comparison between the analytical and numerical approach, elastic moduli

\begin{tabular}{ccccccc}
\hline No. cells & $\ell(\mathrm{mm})$ & $E_{1}^{*}(\mathrm{GPa})$ & $E_{2}^{*}(\mathrm{GPa})$ & $\nu_{12}^{*}$ & $\nu_{21}^{*}$ & $G^{*}(\mathrm{GPa})$ \\
\hline $10 \times 10$ & 5 & 12.50 & 10.00 & 0 & 0 & 0.09 \\
$50 \times 50$ & 1 & 9.57 & 9.18 & 0 & 0 & 0.05 \\
$100 \times 100$ & 0.5 & 9.28 & 9.10 & 0 & 0 & 0.05 \\
$200 \times 200$ & 0.25 & 9.15 & 9.06 & 0 & 0 & 0.05 \\
$250 \times 250$ & 0.2 & 9.12 & 9.05 & 0 & 0 & 0.05 \\
$400 \times 400$ & 0.125 & 9.08 & 9.04 & 0 & 0 & 0.05 \\
$500 \times 500$ & 0.1 & 9.08 & 9.04 & 0 & 0 & 0.05 \\
Analytical results & & 9.00 & 9.00 & 0 & 0 & 0.05 \\
\hline
\end{tabular}


The area of the unit cell is $A_{0}=\sqrt{3} \ell^{2} / 2$, with $\ell$ the length of the beams [9], [4]. As before, the elastic energy of each beam is obtained by superposition principle

$$
w^{e}=\frac{1}{2}\left(\mathbf{u}^{e}\right)^{T} \cdot \mathbf{k}_{b}^{e} \mathbf{u}^{e}+\frac{1}{2}\left(\frac{1}{2}\left(\boldsymbol{\Delta} \mathbf{u}^{e, a}\right)^{T} \cdot \mathbf{k}_{w f}^{e} \boldsymbol{\Delta} \mathbf{u}^{e, a}\right)+\frac{1}{2}\left(\frac{1}{2}\left(\boldsymbol{\Delta} \mathbf{u}^{e, b}\right)^{T} \cdot \mathbf{k}_{w f}^{e} \boldsymbol{\Delta} \mathbf{u}^{e, b}\right)
$$

with $\mathbf{u}^{e}, \boldsymbol{\Delta} \mathbf{u}^{e, a}, \boldsymbol{\Delta} \mathbf{u}^{e, b}, \mathbf{k}_{b}^{e}, \mathbf{k}_{w f}$, in turn, the vector of nodal displacements, the elongation of the two sets of springs and the stiffness matrices previously defined. See Appendix $\mathrm{E}$ for further details. The elastic energy of the unit cell, $W$, derives from that of the six beams it consists of. Note that each beam is shared between two adjacent cells. Consequently, each member contributes only half of its strain energy to the unit cell.

It is not difficult to see that the first node of each beam coincides with the central node (0). So, denoted by $\mathbf{u}_{0}$ the displacements of the node (0) and by $\boldsymbol{\Delta} \mathbf{u}_{0}^{a}, \boldsymbol{\Delta} \mathbf{u}_{0}^{b}$ the elongation of the springs in (0), follows $\mathbf{u}_{i}=\mathbf{u}_{0}, \boldsymbol{\Delta} \mathbf{u}_{i}^{a}=\boldsymbol{\Delta} \mathbf{u}_{0}^{a}$ and $\boldsymbol{\Delta} \mathbf{u}_{i}^{b}=\boldsymbol{\Delta} \mathbf{u}_{0}^{b}$.

As done in [7], [1], expressing (C.2) in the global reference, adding up forces at the central node (0) and condensing the corresponding degrees of freedom to take account of the forces balance in $(0)$, leads to

$$
W=W\left(\mathbf{u}_{j}, \boldsymbol{\Delta} \mathbf{u}_{j}^{a}, \boldsymbol{\Delta} \mathbf{u}_{j}^{b}\right), \quad j=1,2,3,4,5,6 .
$$
by

The assumption that in the limit $\ell \rightarrow 0$ the discrete variables $\left(\mathbf{u}_{\mathbf{j}}, \varphi_{j}\right)$ can be expressed

$$
\mathbf{u}_{j}=\hat{\mathbf{u}}_{0}+\nabla \hat{\mathbf{u}} \mathbf{b}_{j}, \quad \varphi_{j}=\hat{\varphi}_{0}+\nabla \hat{\varphi} \mathbf{b}_{j}, \quad j=1,2,3,4,5,6
$$

provides the continuum description of the discrete structure. The terms $\hat{\mathbf{u}}_{0}$ and $\hat{\varphi}_{0}$ in (C.4) are the values of $\hat{\mathbf{u}}(\cdot)$ and $\hat{\varphi}(\cdot)$ at the central point of the cell in the continuum description. Substituting (C.4) into (C.3) gives the strain energy of the unit cell as a function of the fields $\hat{\mathbf{u}}$ and $\hat{\varphi}$.

Finally, dividing the expression that turns out from the calculation by the area of the unit cell, $A_{0}$, gives the strain energy density in the continuum approximation $w$ :

$$
\begin{aligned}
w= & \frac{\sqrt{3}\left(C_{\ell}^{2} \ell^{4}\left(3 \varepsilon_{11}^{2}+4 \varepsilon_{12}^{2}+2 \varepsilon_{11} \varepsilon_{22}+3 \varepsilon_{22}^{2}\right)+48 C_{\ell} D_{\ell} \ell^{2}\left(\varepsilon_{11}^{2}+\varepsilon_{22}^{2}+2 \varepsilon_{12}^{2}\right)\right)}{96 D_{\ell} \ell^{3}+8 C_{\ell} \ell^{5}}+ \\
& \frac{6 \sqrt{3}\left(D_{\ell}^{2}\left(3 \varepsilon_{11}^{2}+12 \varepsilon_{12}^{2}-6 \varepsilon_{11} \varepsilon_{22}+3 \varepsilon_{22}^{2}\right)+D_{\ell}\left(12 D_{\ell}+C_{\ell} \ell^{2}\right)(\omega-\hat{\varphi})^{2}\right)}{12 D_{\ell} \ell^{3}+C_{\ell} \ell^{5}}+ \\
& \frac{2 \sqrt{3}\left(D_{\ell} \ell^{2}\left(3 D_{\ell}+C_{\ell} \ell^{2}\right)\left(\hat{\varphi}_{, 1}^{2}+\hat{\varphi}_{, 2}^{2}\right)\right)}{12 D_{\ell} \ell^{3}+C_{\ell} \ell^{5}}+ \\
& \frac{K_{w}\left(39\left(59 \varepsilon_{11}^{2}+96 \varepsilon_{12}^{2}+22 \varepsilon_{11} \varepsilon_{22}+59 \varepsilon_{22}^{2}\right)+70 \ell^{2}\left(\hat{\varphi}_{, 1}^{2}+\hat{\varphi}_{, 2}^{2}\right)\right)}{4480 \sqrt{3}}
\end{aligned}
$$

been $C_{\ell}=\frac{E_{s} h}{1-\nu_{s}^{2}}$ and $D_{\ell}=\frac{E_{s} h^{3}}{12\left(1-\nu_{s}^{2}\right)}$, respectively, the tensile and bending stiffness (per unit width) of the beams, $h$ the thickness, $E_{s}$ and $\nu_{s}$ the Young's modulus and the Poisson's ratio of the cell walls material, $K_{w}=k_{w} \ell, k_{w}$ the Winkler foundation constant per unit width. In particular, the resulting energy density

$$
w=w\left(\varepsilon_{\alpha \beta},(\omega-\hat{\varphi}), \hat{\varphi}, \alpha\right)
$$


is a function of the infinitesimal strains $\varepsilon_{\alpha \beta}=\frac{1}{2}\left(\hat{u}_{\alpha, \beta}+\hat{u}_{\beta, \alpha}\right)$ and the infinitesimal rotation $\omega=\frac{1}{2}\left(\hat{u}_{1,2}-\hat{u}_{2,1}\right)$ that represent, respectively, the symmetric and skew-symmetric part of $\nabla \hat{\mathbf{u}}$, as in the classical continuum mechanics, and of the microrotation gradients, $\hat{\varphi}, \alpha$.

After rewriting (C.5) in terms of $c \equiv C_{\ell} / \ell=\frac{E_{s}(h / \ell)}{1-\nu_{s}^{2}}$ and $d \equiv D_{\ell} / \ell^{3}=\frac{E_{s}(h / \ell)^{3}}{12\left(1-\nu_{s}^{2}\right)}$, it emerges, as before, that the coefficients are independent of $\ell$, with the exception of the microrotation gradients that scale with first order in $\ell$. Consequently, in the limit $\ell \rightarrow 0$ the contribution of the microrotation gradients is missing and the equivalent continuum is non-polar. Accordingly, the strain energy density in the continuum approximation takes the form:

$$
\begin{aligned}
w= & \frac{\sqrt{3}\left(C_{\ell}^{2} \ell^{4}\left(3 \varepsilon_{11}^{2}+4 \varepsilon_{12}^{2}+2 \varepsilon_{11} \varepsilon_{22}+3 \varepsilon_{22}^{2}\right)+48 C_{\ell} D_{\ell} \ell^{2}\left(\varepsilon_{11}^{2}+\varepsilon_{22}^{2}+2 \varepsilon_{12}^{2}\right)\right)}{96 D_{\ell} \ell^{3}+8 C_{\ell} \ell^{5}}+ \\
& \frac{6 \sqrt{3}\left(D_{\ell}^{2}\left(3 \varepsilon_{11}^{2}+12 \varepsilon_{12}^{2}-6 \varepsilon_{11} \varepsilon_{22}+3 \varepsilon_{22}^{2}\right)+D_{\ell}\left(12 D_{\ell}+C_{\ell} \ell^{2}\right)(\omega-\hat{\varphi})^{2}\right)}{12 D_{\ell} \ell^{3}+C_{\ell} \ell^{5}}+ \\
& \frac{K_{w}\left(39\left(59 \varepsilon_{11}^{2}+96 \varepsilon_{12}^{2}+22 \varepsilon_{11} \varepsilon_{22}+59 \varepsilon_{22}^{2}\right)\right)}{4480 \sqrt{3}}
\end{aligned}
$$

\section{Appendix C.2. Constitutive equations}

The constitutive equations ensue from (C.7):

$$
\begin{aligned}
& \sigma_{11}=\sigma_{11}^{s y m}=\left(\frac{\sqrt{3}\left(3 C_{\ell} \ell^{2}+12 D_{\ell}\right)}{4 \ell^{3}}+\frac{767 K_{w}}{2240 \sqrt{3}}\right) \varepsilon_{11}+\left(\frac{\sqrt{3}\left(C_{\ell} \ell^{2}-12 D_{\ell}\right)}{4 \ell^{3}}+\frac{143 K_{w}}{2240 \sqrt{3}}\right) \varepsilon_{22}, \\
& \sigma_{22}=\sigma_{22}^{s y m}=\left(\frac{\sqrt{3}\left(3 C_{\ell} \ell^{2}+12 D_{\ell}\right)}{4 \ell^{3}}+\frac{767 K_{w}}{2240 \sqrt{3}}\right) \varepsilon_{22}+\left(\frac{\sqrt{3}\left(C_{\ell} \ell^{2}-12 D_{\ell}\right)}{4 \ell^{3}}+\frac{143 K_{w}}{2240 \sqrt{3}}\right) \varepsilon_{11}, \\
& \sigma_{12}^{s y m}=\sigma_{21}^{s y m}=\left(\frac{\sqrt{3}\left(C_{\ell} \ell^{2}+12 D_{\ell}\right)}{2 \ell^{3}}+\frac{39 K_{w}}{140 \sqrt{3}}\right) \varepsilon_{12} \\
& \sigma_{12}^{\text {skw }}=-\sigma_{21}^{\text {skw }}=\frac{12 \sqrt{3} D_{\ell}}{\ell^{3}}(\omega-\hat{\varphi}), \\
& \sigma_{12}=\sigma_{12}^{s y m}+\sigma_{12}^{s k w}, \quad \sigma_{21}=\sigma_{21}^{s y m}+\sigma_{21}^{s k w} . \\
& \sigma_{\gamma \delta}^{\text {sym }} \text { and } \sigma_{\gamma \delta}^{\text {skw }} \text { are, in turn, the symmetric and skew-symmetric part of the not-symmetric } \\
& \text { Cauchy-type stress tensor. }
\end{aligned}
$$

\section{Appendix C.3. Elastic constants}

Let us consider the stress state $\sigma_{11} \neq 0, \sigma_{22}=\sigma_{12}=\sigma_{21}=0$. From (C.8) and Hooke's law, $\sigma_{11}^{\text {sym }}=E_{1}^{*} \varepsilon_{11}$, the Young's modulus in the $\mathbf{e}_{\mathbf{1}}$ direction is:

$$
E_{1}^{*}=\frac{\sigma_{11}}{\varepsilon_{11}}=\frac{\sqrt{3}\left(13 K_{w}\left(1-\nu_{s}^{2}\right)+32 E_{s} \lambda\right)\left(39 K_{w}\left(1-\nu_{s}^{2}\right)+70 E_{s} \lambda\left(1+\lambda^{2}\right)\right)}{2\left(1-\nu_{s}^{2}\right)\left(767 K_{w}\left(1-\nu_{s}^{2}\right)+560 E_{s} \lambda\left(3+\lambda^{2}\right)\right)},
$$




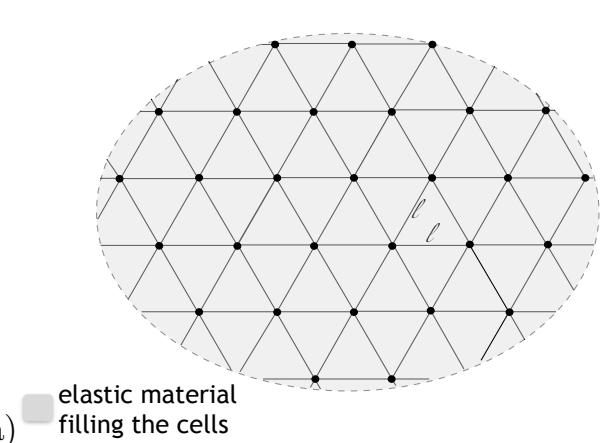

(a) filling the cells

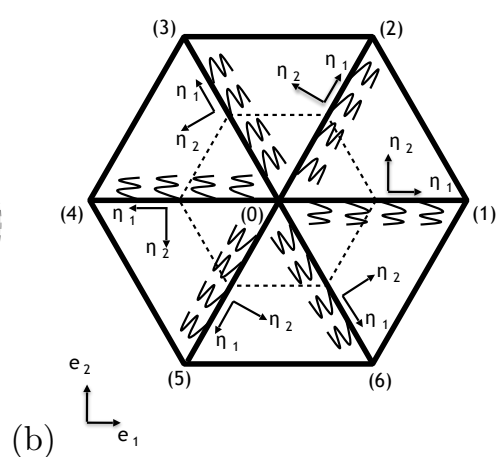

Figure C.4: (a) The equilateral triangular microstructure, (b) The unit cell.
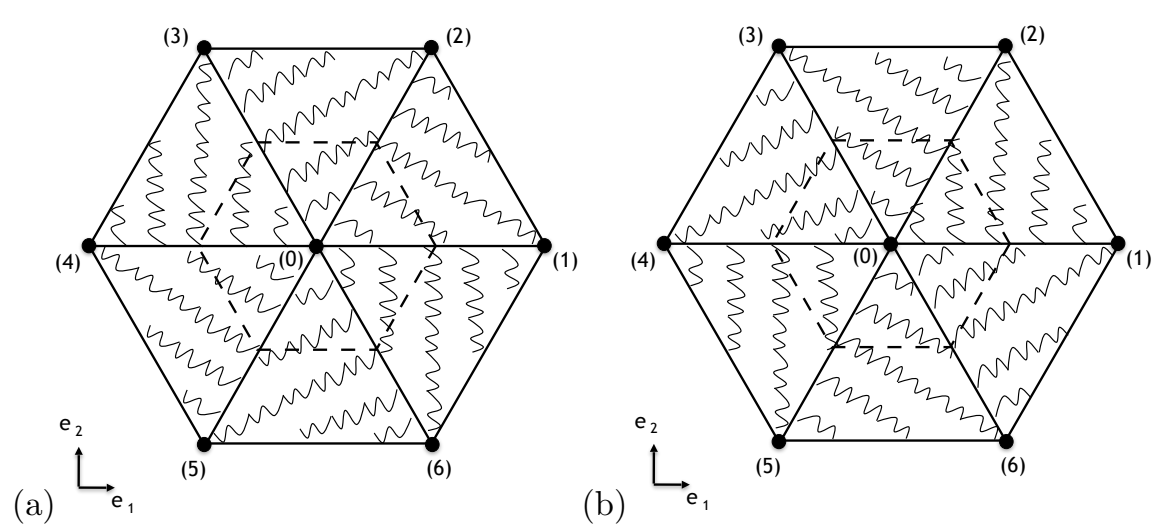

Figure C.5: Equilateral triangular microstructure: (a) Springs a, (b) Springs b. 
while the related Poisson's ratio $\nu_{12}^{*}=-\varepsilon_{22} / \varepsilon_{11}$ is:

$$
\nu_{12}^{*}=\frac{143 K_{w}\left(1-\nu_{s}^{2}\right)-560_{s}\left(\lambda^{2}-1\right)}{767 K_{w}\left(1-\nu_{s}^{2}\right)+560_{s}\left(\lambda^{2}+3\right)} .
$$

Similarly, the stress state $\sigma_{22} \neq 0, \sigma_{11}=\sigma_{12}=\sigma_{21}=0$ leads to the Young's modulus in the $\mathbf{e}_{2}$ direction, $E_{2}^{*}=E_{1}^{*} \equiv E^{*}$, and to the related Poisson's ratio, $\nu_{21}^{*}=-\varepsilon_{11} / \varepsilon_{22}=$ $\nu_{12}^{*} \equiv \nu^{*}$.

Finally, the stress state $\sigma_{12}^{\text {sym }} \neq 0, \sigma_{11}=\sigma_{22}=0$ yields the tangential elastic modulus, $G^{*}=\sigma_{12}^{\text {sym }} / 2 \varepsilon_{12}$ :

$$
G^{*}=\frac{\sqrt{3}\left(39 K_{w}\left(1-\nu_{s}^{2}\right)+70_{s}\left(\lambda^{2}+1\right)\right)}{280\left(1-\nu_{s}^{2}\right)} .
$$

Appendix C.4. Comparison between the analytical and numerical approach

In terms of stress, the compact expression of the constitutive equations derived in Section C.2 is

$$
\left[\begin{array}{l}
\varepsilon_{11} \\
\varepsilon_{22} \\
\varepsilon_{12}
\end{array}\right]=\left[\begin{array}{lll}
C_{11}^{*} & C_{12}^{*} & C_{13}^{*} \\
C_{21}^{*} & C_{22}^{*} & C_{23}^{*} \\
C_{31}^{*} & C_{32}^{*} & C_{33}^{*}
\end{array}\right]\left[\begin{array}{l}
\sigma_{11}^{s y m} \\
\sigma_{22}^{\text {sym }} \\
\sigma_{12}^{\text {sym }}
\end{array}\right],
$$

with

$$
\begin{array}{cll}
C_{11}^{*}=C_{22}^{*} & =\frac{C_{22} C_{33}}{C_{22}^{2} C_{33}-C_{12}^{2} C_{33}}, & C_{12}^{*}=C_{21}^{*}=\frac{C_{12} C_{33}}{C_{22}^{2} C_{33}-C_{12}^{2} C_{33}}, \\
C_{33}^{*} & =\frac{C_{22}^{2}-C_{12}^{2}}{C_{22}^{2} C_{33}-C_{12}^{2} C_{33}}, & C_{13}^{*}=C_{23}^{*}=C_{31}^{*}=C_{32}^{*}=0 .
\end{array}
$$

and

$$
\begin{aligned}
C_{11}=C_{22} & =\frac{\sqrt{3}\left(3 C_{\ell} \ell^{2}+12 D_{\ell}\right)}{4 \ell^{3}}+\frac{767 K_{w}}{2240 \sqrt{3}}=\frac{\sqrt{3} E_{s} \lambda\left(3+\lambda^{2}\right)}{4\left(1-\nu_{s}^{2}\right)}+\frac{767 K_{w}}{2240 \sqrt{3}} \\
C_{12}=C_{21} & =\frac{\sqrt{3}\left(C_{\ell} \ell^{2}-12 D_{\ell}\right)}{4 \ell^{3}}+\frac{143 K_{w}}{2240 \sqrt{3}}=\frac{\sqrt{3} E_{s} \lambda\left(3-\lambda^{2}\right)}{4\left(1-\nu_{s}^{2}\right)}+\frac{143 K_{w}}{2240 \sqrt{3}} \\
C_{33} & =\frac{\sqrt{3}\left(C_{\ell} \ell^{2}+12 D_{\ell}\right)}{2 \ell^{3}}+\frac{39 K_{w}}{140 \sqrt{3}}=\frac{\sqrt{3} E_{s} \lambda^{3}}{\left(1-\nu_{s}^{2}\right)} \\
C_{13}=C_{23}= & C_{31}=C_{32}=0 .
\end{aligned}
$$

As in Appendix B, finite element simulations assess the analytical model. Specifically, the Euler-Bernoulli beam on Winkler foundation elements model the composite microstructure. The cell wall material, assumed to be isotropic linear elastic, has Young's modulus $E_{s}=79 \mathrm{GPa}$, Poisson's ratio $\nu_{s}=0.35$ and thickness $h=0.1 \ell$, while $K_{w}=10^{-2} E_{s}$. The numerical analysis involve a $75 \times 50 \mathrm{~mm}$ rectangular domain discretized in an increasing number of equilateral triangular cells having gradually smaller length $\ell$. As in Appendix B, the load conditions are the uniaxial compression, uniaxial traction and in-plane shear. Forces of the same intensity acting at the boundary, unconstrained nodes of the domain simulate the loading states. Again, the corresponding 
Table C.3: Equilateral triangular microstructure: comparison between the analytical and numerical approach, $C_{i j}^{*}$ constants

\begin{tabular}{cccccccc}
\hline No. cells & $\ell(\mathrm{mm})$ & $C_{11}^{*}$ & $C_{22}^{*}$ & $C_{12}^{*}$ & $C_{21}^{*}$ & $C_{33}^{*}$ & $C_{13}^{*}=C_{23}^{*}=C_{31}^{*}=C_{32}^{*}$ \\
\hline $15 \times 10$ & 5 & 6.05 & 6.08 & 6.02 & 6.03 & 7.50 & 0 \\
$75 \times 50$ & 1 & 6.13 & 6.13 & 6.03 & 6.03 & 5.90 & 0 \\
$100 \times 100$ & 0.5 & 6.25 & 6.23 & 6.03 & 6.05 & 5.90 & 0 \\
$300 \times 200$ & 0.25 & 6.27 & 6.29 & 6.10 & 6.09 & 5.10 & 0 \\
$375 \times 250$ & 0.2 & 6.35 & 6.34 & 6.15 & 6.13 & 4.80 & 0 \\
$600 \times 400$ & 0.125 & 6.38 & 6.39 & 6.22 & 6.21 & 4.60 & 0 \\
$750 \times 500$ & 0.1 & 6.38 & 6.39 & 6.22 & 6.22 & 4.60 & 0 \\
Analytical results & & 6.43 & 6.43 & 6.40 & 6.40 & 4.50 & 0 \\
\hline
\end{tabular}

Table C.4: Equilateral triangular microstructure: comparison between the analytical and numerical approach, elastic moduli

\begin{tabular}{ccccccc}
\hline No. cells & $\ell(\mathrm{mm})$ & $E_{1}^{*}(\mathrm{GPa})$ & $E_{2}^{*}(\mathrm{GPa})$ & $\nu_{12}^{*}$ & $\nu_{21}^{*}$ & $G^{*}(\mathrm{GPa})$ \\
\hline $15 \times 10$ & 5 & 11.33 & 11.48 & 0.42 & 0.45 & 4.70 \\
$75 \times 50$ & 1 & 11.33 & 11.39 & 0.41 & 0.40 & 4.63 \\
$100 \times 100$ & 0.5 & 11.10 & 11.08 & 0.41 & 0.40 & 4.63 \\
$300 \times 200$ & 0.25 & 10.91 & 10.90 & 0.32 & 0.33 & 4.51 \\
$375 \times 250$ & 0.2 & 10.70 & 10.78 & 0.33 & 0.33 & 4.50 \\
$600 \times 400$ & 0.125 & 10.61 & 10.50 & 0.33 & 0.33 & 4.22 \\
$750 \times 500$ & 0.1 & 10.50 & 10.47 & 0.33 & 0.33 & 4.10 \\
Analytical results & & 10.46 & 10.46 & 0.33 & 0.33 & 3.94 \\
\hline
\end{tabular}

effective stiffness components are calculated as the ratio between the average volume strain,

$$
\bar{\varepsilon}_{i j}=\frac{1}{V} \int_{V} \varepsilon_{i j} d V, \quad i, j=1,2,
$$

and the applied stress (see Appendix B).

The results of the analysis are presented in Tables (C.3) and (C.4). In Table (C.3) the analytical and numerical values of the $C_{i j}^{*}$ constants are compared, while Table (C.4) deals with the elastic constants. As it can be seen, the results from the continuum formulation compare reasonably well with the numerical solutions.

\section{Appendix D. The composite cellular material with square microstructure: focus on springs}

As Figure (D.6) shows, the elongation of the elastic springs is expressed by

- Beam (0)-(1) 
(a)
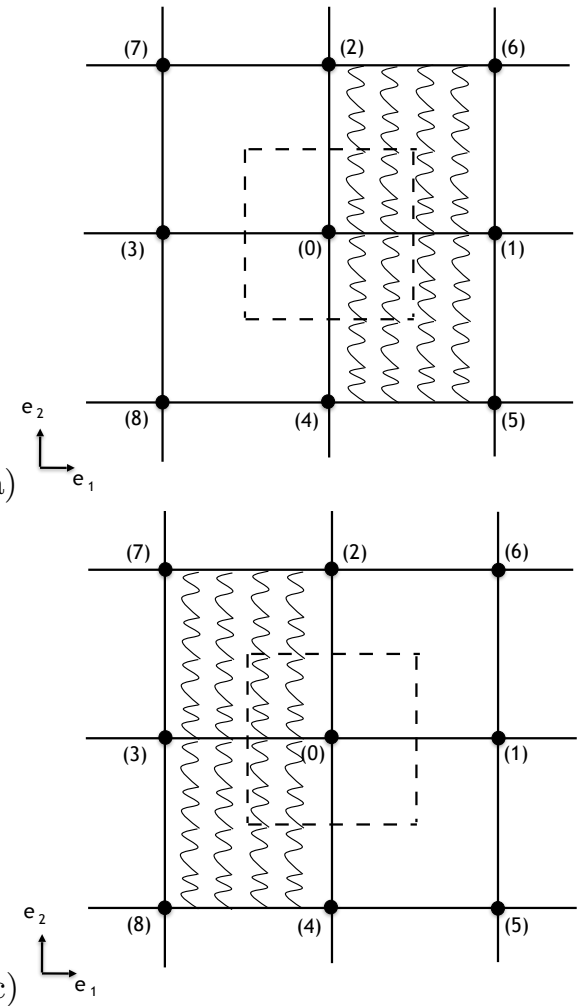

(b)
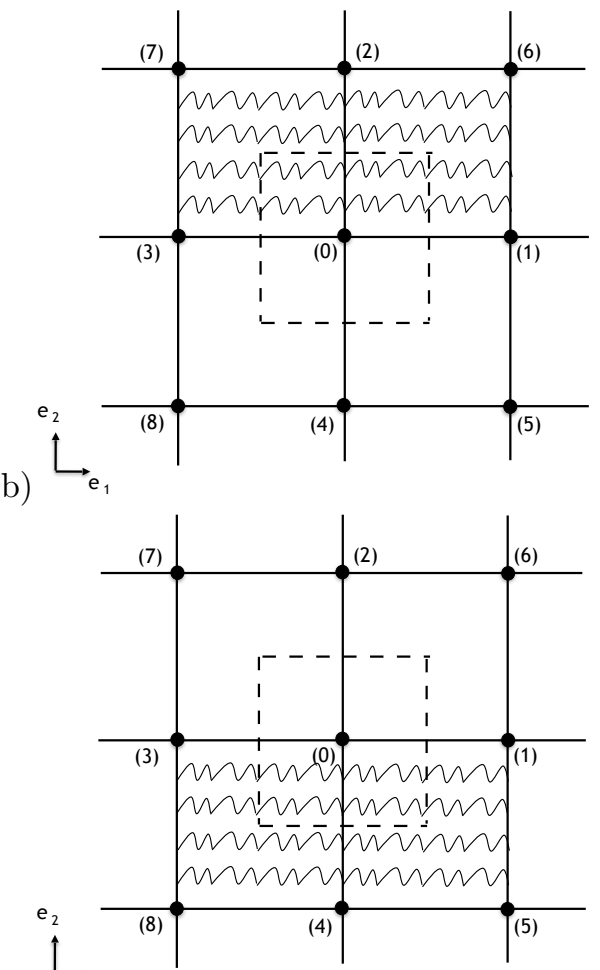

(c) $\rightarrow_{e_{1}}$

Figure D.6: The unit cell with focus on springs in the square microstructure. (a) Beam (0)-(1), (b) Beam (0)-(2), (c) Beam (0)-(3), (d) Beam (0)-(4).

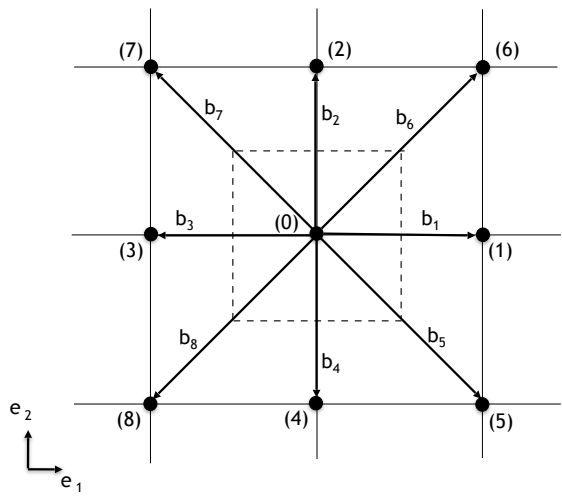

Figure D.7: The $\mathbf{b}_{i}$ vectors in the square microstructure. 
In the discrete system

$$
\boldsymbol{\Delta} \mathbf{u}^{1, a}=\left[\begin{array}{c}
\mathbf{u}_{0}-\mathbf{u}_{4} \\
\varphi_{0}-\varphi_{4} \\
\mathbf{u}_{1}-\mathbf{u}_{5} \\
\varphi_{1}-\varphi_{5}
\end{array}\right], \quad \boldsymbol{\Delta} \mathbf{u}^{1, b}=\left[\begin{array}{c}
\mathbf{u}_{0}-\mathbf{u}_{2} \\
\varphi_{0}-\varphi_{2} \\
\mathbf{u}_{1}-\mathbf{u}_{6} \\
\varphi_{1}-\varphi_{6}
\end{array}\right]
$$

while in the continuum description

$$
\begin{gathered}
\mathbf{u}_{0}=\hat{\mathbf{u}}, \quad \varphi_{0}=\hat{\varphi}, \\
\mathbf{u}_{i}=\hat{\mathbf{u}}+\nabla \hat{\mathbf{u}} \mathbf{b}_{i}+\frac{1}{2}\left(\nabla^{2} \hat{\mathbf{u}} \mathbf{b}_{i}^{2}\right), \\
\varphi_{i}=\hat{\varphi}+\nabla \hat{\varphi} \mathbf{b}_{i}+\frac{1}{2}\left(\nabla^{2} \hat{\varphi} \mathbf{b}_{i}^{2}\right), \quad i=1,2,4,5,6 .
\end{gathered}
$$

Substituting (D.2)-(D.10) into (D.1) leads to

$$
\begin{gathered}
\Delta \mathbf{u}^{1, a}=\left[\begin{array}{c}
-\nabla \hat{\mathbf{u}} \mathbf{b}_{4}-\left(\nabla^{2} \hat{\mathbf{u}} \mathbf{b}_{4}^{2}\right) / 2 \\
-\nabla \hat{\varphi} \mathbf{b}_{4}-\left(\nabla^{2} \hat{\varphi} \mathbf{b}_{4}^{2}\right) / 2 \\
\nabla \hat{\mathbf{u}} \mathbf{b}_{1}+\left(\nabla^{2} \hat{\mathbf{u}} \mathbf{b}_{1}^{2}\right) / 2-\nabla \hat{\mathbf{u}} \mathbf{b}_{5}-\left(\nabla^{2} \hat{\mathbf{u}} \mathbf{b}_{5}^{2}\right) / 2 \\
\nabla \hat{\varphi} \mathbf{b}_{1}+\left(\nabla^{2} \hat{\varphi} \mathbf{b}_{1}^{2}\right) / 2-\nabla \hat{\varphi} \mathbf{b}_{5}-\left(\nabla^{2} \hat{\varphi} \mathbf{b}_{5}^{2}\right) / 2
\end{array}\right], \\
\Delta \mathbf{u}^{1, b}=\left[\begin{array}{c}
-\nabla \hat{\mathbf{u}} \mathbf{b}_{2}-\left(\nabla^{2} \hat{\mathbf{u}} \mathbf{b}_{2}^{2}\right) / 2 \\
-\nabla \hat{\varphi} \mathbf{b}_{2}-\left(\nabla^{2} \hat{\varphi} \mathbf{b}_{2}^{2}\right) / 2 \\
\nabla \hat{\mathbf{u}} \mathbf{b}_{1}+\left(\nabla^{2} \hat{\mathbf{u}} \mathbf{b}_{1}^{2}\right) / 2-\nabla \hat{\mathbf{u}} \mathbf{b}_{6}-\left(\nabla^{2} \hat{\mathbf{u}} \mathbf{b}_{6}^{2}\right) / 2 \\
\nabla \hat{\varphi} \mathbf{b}_{1}+\left(\nabla^{2} \hat{\varphi} \mathbf{b}_{1}^{2}\right) / 2-\nabla \hat{\varphi} \mathbf{b}_{6}-\left(\nabla^{2} \hat{\varphi} \mathbf{b}_{6}^{2}\right) / 2
\end{array}\right] .
\end{gathered}
$$

Similarly, for the beams (0)-(2), (0)-(3), (0)-(4):

- Beam (0)- $(\mathrm{j})$

Discrete system

$$
\boldsymbol{\Delta} \mathbf{u}^{j, a}=\left[\begin{array}{c}
\mathbf{u}_{0}-\mathbf{u}_{k} \\
\varphi_{0}-\varphi_{k} \\
\mathbf{u}_{2}-\mathbf{u}_{l} \\
\varphi_{2}-\varphi_{l}
\end{array}\right], \quad \boldsymbol{\Delta} \mathbf{u}^{j, b}=\left[\begin{array}{c}
\mathbf{u}_{0}-\mathbf{u}_{m} \\
\varphi_{0}-\varphi_{m} \\
\mathbf{u}_{2}-\mathbf{u}_{n} \\
\varphi_{2}-\varphi_{n}
\end{array}\right]
$$

Continuum description

$$
\begin{gathered}
\mathbf{u}_{0}=\hat{\mathbf{u}}, \quad \varphi_{0}=\hat{\varphi} \\
\mathbf{u}_{i}=\hat{\mathbf{u}}+\nabla \hat{\mathbf{u}} \mathbf{b}_{i}+\frac{1}{2}\left(\nabla^{2} \hat{\mathbf{u}} \mathbf{b}_{i}^{2}\right), \\
\varphi_{i}=\hat{\varphi}+\nabla \hat{\varphi} \mathbf{b}_{i}+\frac{1}{2}\left(\nabla^{2} \hat{\varphi} \mathbf{b}_{i}^{2}\right), \quad i=j, k, l, m, n
\end{gathered}
$$

and

$$
\boldsymbol{\Delta} \mathbf{u}^{j, a}=\left[\begin{array}{c}
-\nabla \hat{\mathbf{u}} \mathbf{b}_{k}-\left(\nabla^{2} \hat{\mathbf{u}} \mathbf{b}_{k}^{2}\right) / 2 \\
-\nabla \hat{\varphi} \mathbf{b}_{k}-\left(\nabla^{2} \hat{\varphi} \mathbf{b}_{k}^{2}\right) / 2 \\
\nabla \hat{\mathbf{u}} \mathbf{b}_{j}+\left(\nabla^{2} \hat{\mathbf{u}} \mathbf{b}_{j}^{2}\right) / 2-\nabla \hat{\mathbf{u}} \mathbf{b}_{l}-\left(\nabla^{2} \hat{\mathbf{u}} \mathbf{b}_{l}^{2}\right) / 2 \\
\nabla \hat{\varphi} \mathbf{b}_{j}+\left(\nabla^{2} \hat{\varphi} \mathbf{b}_{j}^{2}\right) / 2-\nabla \hat{\varphi} \mathbf{b}_{l}-\left(\nabla^{2} \hat{\varphi} \mathbf{b}_{l}^{2}\right) / 2
\end{array}\right]
$$




$$
\boldsymbol{\Delta} \mathbf{u}^{j, b}=\left[\begin{array}{c}
-\nabla \hat{\mathbf{u}} \mathbf{b}_{m}-\left(\nabla^{2} \hat{\mathbf{u}} \mathbf{b}_{m}^{2}\right) / 2 \\
-\nabla \hat{\varphi} \mathbf{b}_{m}-\left(\nabla^{2} \hat{\varphi} \mathbf{b}_{m}^{2}\right) / 2 \\
\nabla \hat{\mathbf{u}} \mathbf{b}_{j}+\left(\nabla^{2} \hat{\mathbf{u}} \mathbf{b}_{j}^{2}\right) / 2-\nabla \hat{\mathbf{u}} \mathbf{b}_{n}-\left(\nabla^{2} \hat{\mathbf{u}} \mathbf{b}_{n}^{2}\right) / 2 \\
\nabla \hat{\varphi} \mathbf{b}_{j}+\left(\nabla^{2} \hat{\varphi} \mathbf{b}_{j}^{2}\right) / 2-\nabla \hat{\varphi} \mathbf{b}_{n}-\left(\nabla^{2} \hat{\varphi} \mathbf{b}_{n}^{2}\right) / 2
\end{array}\right]
$$

In particular,

Beam (0)-(2): $j=2, k=1, l=6, m=3, n=7$,

Beam (0)-(3): $j=3, k=2, l=7, m=4, n=8$,

Beam (0)-(4): $j=4, k=3, l=8, m=1, n=5$.

Finally, the vectors $\mathbf{b}_{i}$ (Fig. (D.7)) are

$$
\begin{aligned}
\mathbf{b}_{1}=(\ell, 0), & \mathbf{b}_{2}=(0, \ell), & \mathbf{b}_{3}=(-\ell, 0), & \mathbf{b}_{4}=(0,-\ell), \\
\mathbf{b}_{5}=(\ell,-\ell), & \mathbf{b}_{6}=(\ell, \ell), & \mathbf{b}_{7}=(-\ell, \ell), & \mathbf{b}_{8}=(-\ell,-\ell) .
\end{aligned}
$$

\section{Appendix E. Composite cellular material with equilateral triangular microstruc- ture: focus on springs}

In the case of equilateral triangular microstructure, the elongation of the springs takes the form (Fig. (E.8), (E.9))

- Beam (0)-(1)

Discrete system

$$
\boldsymbol{\Delta} \mathbf{u}_{1}^{a}=\left[\begin{array}{c}
\mathbf{u}_{1}-\mathbf{u}_{6} \\
\varphi_{1}-\varphi_{6}
\end{array}\right], \quad \boldsymbol{\Delta} \mathbf{u}_{1}^{b}=\left[\begin{array}{c}
\mathbf{u}_{1}-\mathbf{u}_{2} \\
\varphi_{1}-\varphi_{2}
\end{array}\right] .
$$

In the continuum description,

$$
\mathbf{u}_{i}=\hat{\mathbf{u}}+\nabla \hat{\mathbf{u}} \mathbf{b}_{i}, \quad \varphi_{i}=\hat{\varphi}+\nabla \hat{\varphi} \mathbf{b}_{i}, \quad i=1,6,2
$$

that, substituted in (E.1), lead to

$$
\boldsymbol{\Delta} \mathbf{u}_{1}^{a}=\left[\begin{array}{c}
\nabla \hat{\mathbf{u}} \mathbf{b}_{1}-\nabla \hat{\mathbf{u}} \mathbf{b}_{6} \\
\nabla \hat{\varphi} \mathbf{b}_{1}-\nabla \hat{\varphi} \mathbf{b}_{6}
\end{array}\right], \quad \boldsymbol{\Delta} \mathbf{u}_{1}^{b}=\left[\begin{array}{c}
\nabla \hat{\mathbf{u}} \mathbf{b}_{1}-\nabla \hat{\mathbf{u}} \mathbf{b}_{2} \\
\nabla \hat{\varphi} \mathbf{b}_{1}-\nabla \hat{\varphi} \mathbf{b}_{2}
\end{array}\right] .
$$

For the other beams, similar calculations provide

- Beam (0)-(j)

Discrete system

$$
\boldsymbol{\Delta} \mathbf{u}_{j}^{a}=\left[\begin{array}{c}
\mathbf{u}_{j}-\mathbf{u}_{k} \\
\varphi_{j}-\varphi_{k}
\end{array}\right], \quad \boldsymbol{\Delta} \mathbf{u}_{j}^{b}=\left[\begin{array}{c}
\mathbf{u}_{j}-\mathbf{u}_{l} \\
\varphi_{j}-\varphi_{l}
\end{array}\right] .
$$

Continuum description

$$
\mathbf{u}_{i}=\hat{\mathbf{u}}+\nabla \hat{\mathbf{u}} \mathbf{b}_{i}, \quad \varphi_{i}=\hat{\varphi}+\nabla \hat{\varphi} \mathbf{b}_{i}, \quad i=j, k, l
$$

and

$$
\boldsymbol{\Delta} \mathbf{u}_{j}^{a}=\left[\begin{array}{c}
\nabla \hat{\mathbf{u}} \mathbf{b}_{j}-\nabla \hat{\mathbf{u}} \mathbf{b}_{k} \\
\nabla \hat{\varphi} \mathbf{b}_{j}-\nabla \hat{\varphi} \mathbf{b}_{k}
\end{array}\right], \quad \boldsymbol{\Delta} \mathbf{u}_{j}^{b}=\left[\begin{array}{c}
\nabla \hat{\mathbf{u}} \mathbf{b}_{j}-\nabla \hat{\mathbf{u}} \mathbf{b}_{l} \\
\nabla \hat{\varphi} \mathbf{b}_{j}-\nabla \hat{\varphi} \mathbf{b}_{l}
\end{array}\right],
$$

with 

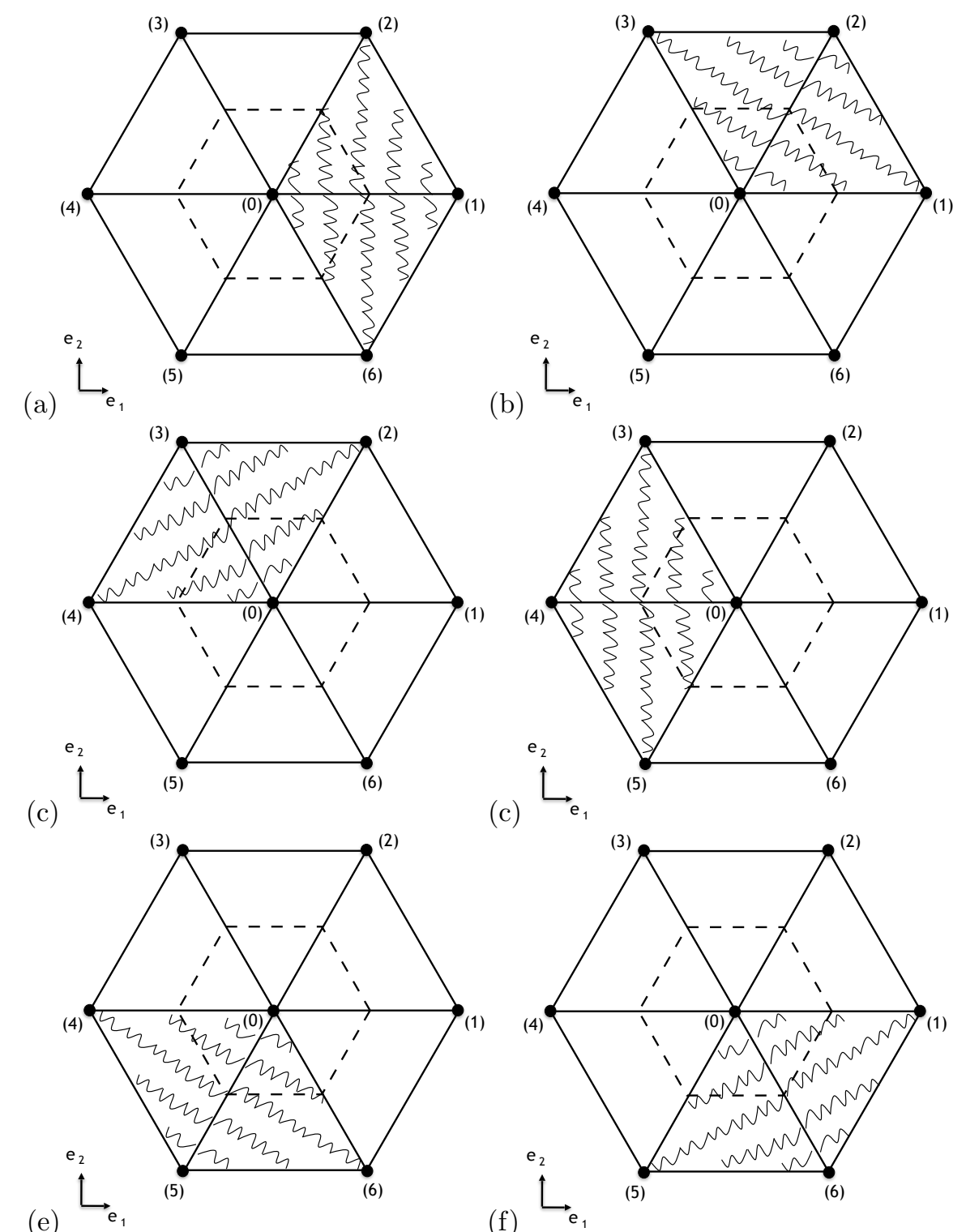

(c) $\mathrm{e}_{1}$

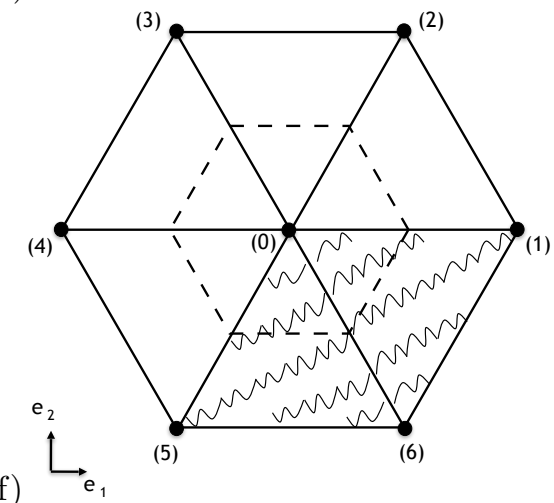

Figure E.8: The unit cell with focus on springs in the equilateral triangular microstructure. (a) Beam (0)-(1), (b) Beam (0)-(2), (c) Beam (0)-(3), (d) Beam (0)-(4), (e) Beam (0)-(5), (f) Beam (0)-(6). 


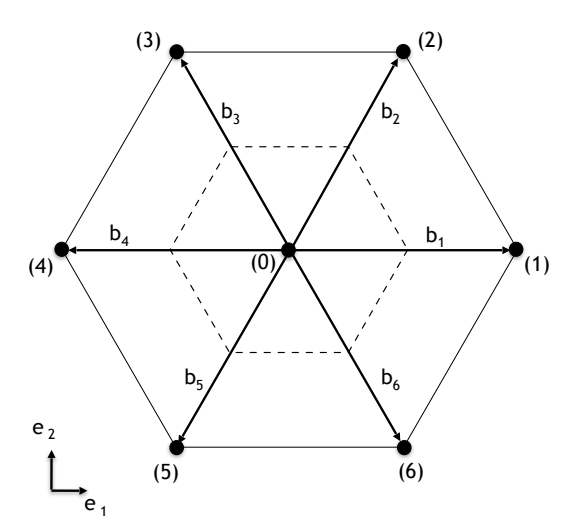

Figure E.9: The $\mathbf{b}_{i}$ vectors in the equilateral triangular microstructure.

Beam (0)-(2): $j=2, k=1, l=3$,

Beam (0)-(3): $j=3, k=2, l=4$,

Beam (0)-(4): $j=4, k=3, l=5$,

Beam (0)-(5): $j=5, k=4, l=6$,

Beam (0)-(6): $j=6, k=5, l=1$.

Finally, as stated, note that each beam is shared between two adjacent beams. So, each member contributes only half of its strain energy to the representative cell.

\section{References}

[1] Ongaro, F., De Falco, P., Pugno, N. M., Barbieri, E., Mechanics of filled cellular materials, Mech. Mater., vol. 97, pp. 26-47, 2016.

[2] Gibson, L. J., Ashby, M. F., Harley, B. A., Cellular Materials in Nature and Medicine, Cambridge University Press, 2010.

[3] Janco, R, Solution Methods for Beam and Frames on Elastic Foundation Using the Finite Element Method, Mechanical Structures and Foundation Engineering, International scientific conference MSFE 2010

[4] Kumar, R. S., McDowell, D. L., Generalized continuum modeling of 2-D periodic cellular solids, Int. J. Solids Struct., vol. 41, pp. 7399-7422, 2004.

[5] Bazant, Z. P., Micropolar medium as model for buckling of grid frameworks, Proceedings of the 12th Midwestern Mechanics Conference, vol. 6, pp. 587-594, 1971.

[6] Bazant, Z. P., Christensen, M., Analogy between micropolar continuum and grid frameworks under initial stress, Int. J. Solids Struct., vol. 8, pp. 327-346, 1972

[7] Davini, C., Ongaro, F., A Homogenized Model for Honeycomb cellular materials, J. Elasticity, vol. 104, pp. 205-226, 2011.

[8] Arabnejad, S., Pasini, D., Mechanical properties of lattice materials via asymptotic homogenization and comparison with alternative homogenization methods, International Journal of Mechanical Sciences, vol. 77, pp. 294-262, 2013.

[9] Chen, J. Y., Huang, Y., Ortiz, M., Fracture analysis of cellular materials: a strain gradient model, J. Mech. Phys. Solids, vol. 46, No. 5, pp. 789-828, 1998. 